\title{
The Genetics of Endophenotypes of Neurofunction to Understand Schizophrenia (GENUS) consortium: A collaborative cognitive and neuroimaging genetics project
}

Gabriëlla A.M. Blokland a,b,c,d, Elisabetta C. del Re ${ }^{\text {ce,f }}$, Raquelle I. Mesholam-Gately c,g , Jorge Jovicich ${ }^{\text {h }}$, Joey W. Trampush ${ }^{\mathrm{i}, \mathrm{j}, \mathrm{k}, \mathrm{l}}$, Matcheri S. Keshavan ${ }^{\mathrm{c}, \mathrm{g}, \mathrm{m}}$, Lynn E. DeLisi ${ }^{\mathrm{c}, \mathrm{e}}$, James T.R. Walters ${ }^{\mathrm{n}}$, Jessica A. Turner ${ }^{\mathrm{o}, \mathrm{p}}$, Anil K. Malhotra ${ }^{\mathrm{i}, \mathrm{j}, \mathrm{k}}$, Todd Lencz ${ }^{\mathrm{i}, \mathrm{j}, \mathrm{k}}$, Martha E. Shenton ${ }^{\mathrm{c}, \mathrm{e}, \mathrm{f}, \mathrm{q}}$, Aristotle N. Voineskos ${ }^{\mathrm{r}, \mathrm{s}}$, Dan Rujescu ${ }^{\mathrm{t}, \mathrm{u}}$, Ina Giegling ${ }^{\mathrm{t}}$, René S. Kahn ${ }^{\mathrm{v}}$, Joshua L. Roffman ${ }^{\mathrm{b}, \mathrm{c}, \mathrm{w}}$, Daphne J. Holt ${ }^{\mathrm{b}, \mathrm{c}, \mathrm{w}}$, Stefan Ehrlich ${ }^{\mathrm{c}, \mathrm{w}, \mathrm{x}}$, Zora Kikinis ${ }^{\mathrm{c}, \mathrm{f}}$, Paola Dazzan ${ }^{\mathrm{y}, \mathrm{z}}$, Robin M. Murray ${ }^{\mathrm{y}, \mathrm{z}}$, Marta Di Forti ${ }^{\mathrm{y}, \mathrm{z}}$, Jimmy Lee ${ }^{\mathrm{aa}}$, Kang Sim ${ }^{\text {aa }}$, Max Lam $^{\text {aa }}$, Rick P.F. Wolthusen ${ }^{c, w, x}$, Sonja M.C. de Zwarte ${ }^{\mathrm{v}}$, Esther Walton ${ }^{\mathrm{v}}$, Donna Cosgrove ${ }^{\mathrm{ab}}$, Sinead Kelly ${ }^{\mathrm{ac}, \mathrm{ad}}$, Nasim Maleki ${ }^{\mathrm{b}, \mathrm{c}, \mathrm{w}}$, Lisa Osiecki ${ }^{\mathrm{a}}$, Marco M. Picchioni ${ }^{\mathrm{y}, \mathrm{z}}$, Elvira Bramon ${ }^{\mathrm{y}, \mathrm{z}, \mathrm{ae}}$, Manuela Russo ${ }^{\mathrm{y}, \mathrm{z}}$, Anthony S. David ${ }^{\mathrm{y}, \mathrm{z}}$, Valeria Mondelli ${ }^{\mathrm{y}, \mathrm{z}}$, Antje A.T.S. Reinders ${ }^{\mathrm{y}, \mathrm{z}}$, M. Aurora Falcone ${ }^{\mathrm{y}, \mathrm{z}}$, Annette M. Hartmann ${ }^{\mathrm{t}}$, Bettina Konte ${ }^{t}$, Derek W. Morris ${ }^{\text {af }}$, Michael Gill ${ }^{\text {ac }}$, Aiden P. Corvin ${ }^{a c}$, Wiepke Cahn ${ }^{v}$, New Fei Ho ${ }^{\text {aa }}$, Jian Jun Liu ${ }^{\text {ag }}$, Richard S.E. Keefe ${ }^{\text {ah }}$, Randy L. Gollub ${ }^{\text {b,c,w }}$, Dara S. Manoach ${ }^{\text {b,c,w }}$, Vince D. Calhoun ${ }^{\text {o,ai }}$, S. Charles Schulz ${ }^{\text {aj }}$, Scott R. Sponheim ${ }^{\text {aj }}$, Donald C. Goff ${ }^{\text {c,ak }}$, Stephen L. Buka ${ }^{\text {al }}$, Sara Cherkerzian ${ }^{\text {am }}$, Heidi W. Thermenos ${ }^{\text {b,c,g }}$, Marek Kubicki ${ }^{\text {c,f,q,w }}$, Paul G. Nestor ${ }^{\mathrm{c}, \mathrm{e}, \mathrm{an}}$, Erin W. Dickie ${ }^{\mathrm{r}}$, Evangelos Vassos ${ }^{\mathrm{y}, \mathrm{z}}$, Simone Ciufolini ${ }^{\mathrm{y}, \mathrm{z}}$, Tiago Reis Marques ${ }^{\mathrm{y}, \mathrm{z}}$, Nicolas A. Crossley ${ }^{\mathrm{y}, \mathrm{z}}$, Shaun M. Purcell ${ }^{\mathrm{c}, \mathrm{d}, \mathrm{ao}, \mathrm{ap}}$, Jordan W. Smoller a,b,c,d , Neeltje E.M. van Haren ${ }^{\text {v }}$, Timothea Toulopoulou ${ }^{\text {y,aq,ar }}$, Gary Donohoe ${ }^{\text {ac,af }}$,

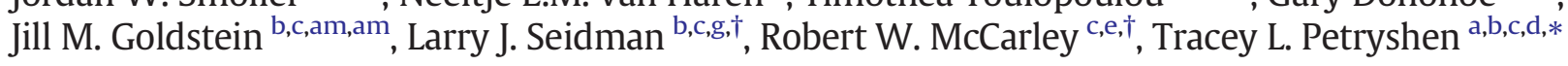

a Psychiatric and Neurodevelopmental Genetics Unit, Center for Genomic Medicine, Massachusetts General Hospital, Boston, MA, United States

b Department of Psychiatry, Massachusetts General Hospital, Boston, MA, United States

c Department of Psychiatry, Harvard Medical School, Boston, MA, United States

d Stanley Center for Psychiatric Research, Broad Institute of MIT and Harvard, Cambridge, MA, United States

e Department of Psychiatry, Veterans Affairs Boston Healthcare System, Brockton, MA, United States

${ }^{\mathrm{f}}$ Psychiatry Neuroimaging Laboratory, Department of Psychiatry, Brigham and Women's Hospital, Boston, MA, United States

g Massachusetts Mental Health Center Public Psychiatry Division, Beth Israel Deaconess Medical Center, Boston, MA, United States

${ }^{\text {h }}$ Center for Mind/Brain Sciences (CiMEC), University of Trento, Trento, Italy

${ }^{i}$ Center for Psychiatric Neuroscience, The Feinstein Institute for Medical Research, Division of Northwell Health, Manhasset, NY, United States

${ }^{j}$ Division of Psychiatry Research, The Zucker Hillside Hospital, Division of Northwell Health, Glen Oaks, NY, United States

${ }^{\mathrm{k}}$ Hofstra Northwell School of Medicine, Departments of Psychiatry and Molecular Medicine, Hempstead, NY, United States

${ }^{1}$ BrainWorkup, LLC, Los Angeles, CA, United States

${ }^{\mathrm{m}}$ University of Pittsburgh Medical Center, Pittsburgh, PA, United States

${ }^{n}$ Department of Psychological Medicine, Cardiff University, Cardiff, United Kingdom

- The Mind Research Network, Albuquerque, NM, United States

${ }^{\mathrm{p}}$ Department of Psychology and Neuroscience Institute, Georgia State University, GA, United States

${ }^{\mathrm{q}}$ Department of Radiology, Brigham and Women's Hospital, Harvard Medical School, Boston, MA, United States

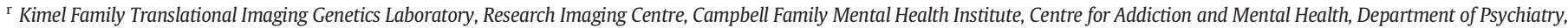

Faculty of Medicine, University of Toronto, Toronto, ON, Canada

${ }^{s}$ Department of Psychiatry and Institute of Medical Science, University of Toronto, Toronto, ON, Canada

${ }^{t}$ Department of Psychiatry, Psychotherapy and Psychosomatics, University of Halle-Wittenberg, Halle, an der Saale, Germany

u Department of Psychiatry, Ludwig Maximilians University, Munich, Germany

v Brain Centre Rudolf Magnus, Department of Psychiatry, University Medical Centre Utrecht, Utrecht, The Netherlands

${ }^{w}$ MGH/HST Athinoula A. Martinos Center for Biomedical Imaging, Massachusetts General Hospital, Charlestown, MA, United States

${ }^{x}$ Division of Psychological \& Social Medicine and Developmental Neurosciences, Faculty of Medicine, Technische Universität Dresden, Dresden, Germany

${ }^{y}$ Institute of Psychiatry, Psychology, and Neuroscience, King's College London, London, United Kingdom

${ }^{\mathrm{z}}$ National Institute for Health Research (NIHR) Mental Health Biomedical Research Centre at South London and Maudsley NHS Foundation Trust, London, United Kingdom

aa Institute of Mental Health, Woodbridge Hospital, Singapore

\footnotetext{
* Corresponding author at: Psychiatric and Neurodevelopmental Genetics Unit, Center for Genomic Medicine, Massachusetts General Hospital, 185 Cambridge Street, Boston, MA 02114, United States.

E-mail address: tpetryshen@mgh.harvard.edu (T.L. Petryshen).

$\dagger$ Deceased
} 
ab The Cognitive Genetics and Cognitive Therapy Group, Department of Psychology, National University of Ireland, Galway, Ireland

ac Neuropsychiatric Genetics Research Group, Department of Psychiatry, Institute of Molecular Medicine, Trinity College Dublin, Dublin, Ireland

ad Laboratory of Neurolmaging, Keck School of Medicine, University of Southern California, Los Angeles, CA, United States

ae Mental Health Neuroscience Research Department, UCL Division of Psychiatry, University College London, United Kingdom

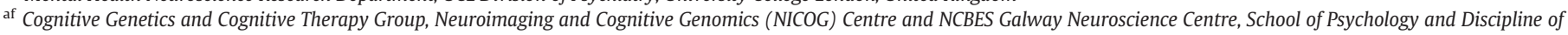
Biochemistry, National University of Ireland, Galway, Ireland

ag Genome Institute, Singapore

ah Department of Psychiatry and Behavioral Sciences, Duke University Medical Center, Durham, NC, United States

ai Department of Electrical and Computer Engineering, University of New Mexico, Albuquerque, NM, United States

aj Department of Psychiatry, University of Minnesota, Minneapolis, MN, United States

ak Nathan S. Kline Institute for Psychiatric Research, Department of Psychiatry, New York University Langone Medical Center, New York, NY, United States

al Department of Epidemiology, Brown University, Providence, RI, United States

am Department of Medicine, Division of Women's Health, Brigham and Women's Hospital, Harvard Medical School, Boston, MA, United States

an Laboratory of Applied Neuropsychology, University of Massachusetts, Boston, MA, United States

ao Department of Psychiatry, Brigham and Women's Hospital, Boston, MA, United States

ap Division of Psychiatric Genomics, Departments of Psychiatry and Genetics and Genomic Sciences, Icahn School of Medicine at Mount Sinai, New York, NY, United States

aq Department of Psychology, Bilkent University, Bilkent, Ankara, Turkey

ar Department of Psychology, The University of Hong Kong, Pokfulam, Hong Kong, SAR, China

\section{A R T I C L E I N F O}

\section{Article history:}

Received 15 June 2017

Received in revised form 15 September 2017

Accepted 20 September 2017

Available online 3 October 2017

\section{Keywords:}

Schizophrenia

Neuropsychology

Cognition

Neuroimaging

MRI

Genetics

\begin{abstract}
A B S T R A C T
Background: Schizophrenia has a large genetic component, and the pathways from genes to illness manifestation are beginning to be identified. The Genetics of Endophenotypes of Neurofunction to Understand Schizophrenia (GENUS) Consortium aims to clarify the role of genetic variation in brain abnormalities underlying schizophrenia. This article describes the GENUS Consortium sample collection.

Methods: We identified existing samples collected for schizophrenia studies consisting of patients, controls, and/ or individuals at familial high-risk (FHR) for schizophrenia. Samples had single nucleotide polymorphism (SNP) array data or genomic DNA, clinical and demographic data, and neuropsychological and/or brain magnetic resonance imaging (MRI) data. Data were subjected to quality control procedures at a central site.

Results: Sixteen research groups contributed data from 5199 psychosis patients, 4877 controls, and 725 FHR individuals. All participants have relevant demographic data and all patients have relevant clinical data. The sex ratio is $56.5 \%$ male and $43.5 \%$ female. Significant differences exist between diagnostic groups for premorbid and current IQ (both $p<1 \times 10^{-10}$ ). Data from a diversity of neuropsychological tests are available for $92 \%$ of participants, and 30\% have structural MRI scans (half also have diffusion-weighted MRI scans). SNP data are available for $76 \%$ of participants. The ancestry composition is 70\% European, 20\% East Asian, $7 \%$ African, and 3\% other. Conclusions: The Consortium is investigating the genetic contribution to brain phenotypes in a schizophrenia sample collection of $>10,000$ participants. The breadth of data across clinical, genetic, neuropsychological, and MRI modalities provides an important opportunity for elucidating the genetic basis of neural processes underlying schizophrenia.
\end{abstract}

(C) 2017 Elsevier B.V. All rights reserved.

\section{Introduction}

Clinical presentation of schizophrenia varies among individuals, but in general is characterized by positive (hallucinations, delusions), negative (social withdrawal), and disorganization symptoms, cognitive impairments, altered brain structure and function, and severe deficits in global and social functioning. There is a generalized cognitive impairment, as well as specific deficits across cognitive domains including processing speed, attention, working memory, verbal memory, and executive functioning, that are present as early as the pre-morbid state during childhood and persist through chronic stages of illness (Lewandowski et al., 2011). There is consistent evidence from schizophrenia neuroimaging studies for ventricular enlargement, reduced gray matter volume of cortical and subcortical brain regions, and reduced white matter volume and fractional anisotropy of predominantly fronto-temporal tracts (Bora et al., 2011; Haijma et al., 2013; Shenton et al., 2001; van Erp et al., 2016). Unaffected relatives of schizophrenia patients exhibit milder cognitive deficits and brain structural abnormalities (Boos et al., 2007; Keshavan et al., 2010; Thermenos et al., 2013), suggesting these abnormalities are risk factors for the disorder rather than secondary effects. The molecular mechanisms underlying these brain abnormalities are only beginning to be unraveled, which has hindered the identification of rational targets for developing better treatments.

A practical approach for elucidating the disease biology is identifying genes that confer risk and characterizing their function within the brain.
It is long known that schizophrenia has a large genetic component, with heritability between 64 and 81\% (Lichtenstein et al., 2009; Sullivan et al., 2003). Genome-wide association studies (GWAS) of schizophrenia case/control datasets by the Psychiatric Genomics Consortium (PGC) have identified over 100 chromosomal loci that have genome-wide significant evidence for association (PGC Schizophrenia Working Group, 2014). GWAS results indicate that schizophrenia is a polygenic disorder, for which thousands of common genetic variants with modest individual effects act in aggregate to increase disease liability (Psychosis Endophenotypes International Consortium et al., 2014; Purcell et al., 2009; Ripke et al., 2013). Rare variants further contribute to schizophrenia liability (CNV and Schizophrenia Working Groups of the Psychiatric Genomics Consortium; Psychosis Endophenotypes International Consortium, 2017; Malhotra and Sebat, 2012).

A promising approach to translate these genetic findings into an understanding of the neural processes involved in schizophrenia is to evaluate their relevance to disease endophenotypes (Gottesman and Gould, 2003). In this context, cognitive measures have a moderate to high heritability $\left(\mathrm{h}^{2}=0.2-0.7\right.$ ) (Seidman et al., 2015; Stone and Seidman, 2016), while volumetric and diffusion brain measures are highly heritable $\left(h^{2}=0.6-0.8\right)$ (Blokland et al., 2012, 2017). Common genetic variation (based on SNPs) explains a substantial proportion of this heritability, estimated at $\mathrm{h}^{2}=0.3-0.4$ for cognitive (Hatzimanolis et al., 2015; Robinson et al., 2015) and brain volume phenotypes (Ge et al., 2015). Moderate to high genetic correlations between schizophrenia and cognitive and brain structural phenotypes $\left(r_{g}=0.5-0.8\right)$ suggest a 
partially shared genetic etiology (Blokland et al., 2017; Bohlken et al., 2016; Lee et al., 2016). Indeed, polygenic risk for schizophrenia is significantly associated with prefrontal inefficiency during working memory performance in patients and controls (Walton et al., 2013a; Walton et al., 2013b), as well as lower cognitive performance among healthy populations (Germine et al., 2016; Hubbard et al., 2016; Lencz et al., 2014; Liebers et al., 2016) and schizophrenia patients (Martin et al., 2015). Specific genetic risk variants have also been associated with altered cognition and brain structure among patients (Donohoe et al., 2010, 2013; Lencz et al., 2010; Martin et al., 2015; Wassink et al., 2012; Yeo et al., 2014) although some studies are negative (van Scheltinga et al., 2013), possibly due to the use of small samples that are prone to inconsistent results. Analyses of large, well-phenotyped samples consisting of both psychosis patients and control individuals will be important for clarifying the role of genetic risk variants in brain abnormalities relevant to illness.

With this in mind, the GENUS Consortium aims to improve knowledge of the contribution of genetic variation to schizophrenia brain abnormalities by investigating relevant brain traits in a large, comprehensively phenotyped sample collection. The GENUS Consortium draws upon the efforts of sixteen research groups that have previously collected samples consisting of psychosis patients (predominantly schizophrenia), unaffected controls, and/or unaffected familial high-risk (FHR) individuals assessed for neuropsychological function and/or brain structure, all of which have genome-wide SNP data or genomic DNA. Assembly of these samples into one harmonized collection substantially increases the statistical power compared to the individual samples alone. The large, well-phenotyped GENUS sample collection provides a prime opportunity to investigate the genetic basis of brain abnormalities in psychosis in order to gain insight into the underlying neural mechanisms. The purpose of this article is to describe the design, composition, and data components of the sample collection, while subsequent articles will focus on data analyses.

\section{Methods}

\subsection{Collection of samples}

Research groups that had previously collected samples for the purpose of schizophrenia studies were identified from the psychiatric genetics community and publications. Criteria for inclusion were: availability of SNP genotype data or genomic DNA, as well as demographic, neuropsychological and/or magnetic resonance imaging (MRI) data, and, for patients, clinical data.

\subsection{Informed consent and ethics approval}

The lead principal investigator for each sample verified approval from their institutional ethics committee for sharing human subject data. All research participants provided written informed consent (or legal guardian consent and subject assent). Ethics approval for the GENUS Consortium study at the central site was obtained from the Partners Healthcare (USA) Institutional Review Board. All data were anonymized prior to transfer to the central site.

\subsection{Clinical and demographic data}

For demographic data, all research groups had collected data on age at recruitment, sex, and education level, and most groups had also collected data on socioeconomic status and handedness. Clinical data were available for patients and, for some samples, FHR individuals. All site-specific clinical variables were renamed according to a common variable naming convention. Raw data underwent quality control analyses at the central site for expected value ranges and outliers. To enable comparison across sites, we computed basic descriptives (means and standard deviations for quantitative variables; frequency tables for categorical variables) and plotted histograms to check for unexpected differences in data distributions. Antipsychotic medication dosages, both current and lifetime, where available, were converted to chlorpromazine equivalents based on published dosage equivalence estimates (Gardner et al., 2010; Woods, 2003).

\subsection{Neuropsychological data}

The specific neuropsychological tests ranged across samples, although all research groups administered tests within the Measurement and Treatment Research to Improve Cognition in Schizophrenia (MATRICS) consensus cognitive battery (Nuechterlein et al., 2008) or tests with similar design and scoring. We therefore focused on MATRICS tests and tests that measure similar cognitive constructs as the MATRICS tests. Additionally, we included visuospatial ability and verbal ability tests, as most groups administered these tests. All site-specific test variables were renamed according to a common variable naming convention. The raw data from each test were checked for errors by calculating descriptive statistics and visualizing data distributions for each study sample. Premorbid IQ was estimated from reading tests (or vocabulary if reading tests were not available), and current IQ from Wechsler Adult Intelligence Scale (WAIS) subtests (see Supplementary Materials).

\subsection{Neuroimaging data}

For those research groups that acquired MRI scans, we required 1.5 or 3 Tesla field strength, and availability of control scans in order to normalize the imaging data. We imposed no restrictions on the scanner vendor or model. As an initial assessment of quality, a subset of 12 scans from each sample ( 3 male patients, 3 female patients, 3 male controls, 3 female controls) were visually inspected for consistent artifacts using 3DSlicer (http://www.slicer.org; Fedorov et al., 2012), including partial brain coverage, wrap-around and motion artifacts, and gross signal/contrast inhomogeneity. Further quality control analyses were carried out upon receipt of the full dataset and will be described elsewhere.

\subsection{SNP genotype data}

Each research group provided raw SNP array genotype data, when available, or genomic DNA extracted from whole blood, buffy coat or saliva ( $\geq 2 \mathrm{ng} / \mu \mathrm{L}$ ) that we genotyped on the Illumina Infinium PsychArray. Although most participants had self-reported ancestry information, we assigned ancestry by merging genotype call data from each sample with the 1000 Genomes Reference Panel (Sudmant et al., 2015; The 1000 Genomes Project Consortium et al., 2015), and applying multidimensional scaling using Plink software (Purcell et al., 2007) to extract ancestry principal components. Model-based clustering ( $\mathrm{R}$ function 'Mclust') was applied to classify participants into ancestral populations as defined by the 1000 Genomes Reference Panel. Basic quality control analyses of raw genotype data consisted of removing unplaced SNPS and confirming consistency between reported sex and X chromosome genotype.

\subsection{Statistical analyses}

Quantitative demographic data from patient, control, and FHR groups were compared using ANOVA. Chi-square tests compared the relative proportions of males/females, ancestral populations, and handedness across groups. For all statistical tests, an uncorrected alpha of 0.05 was applied. 


\section{Results}

\subsection{Central data management}

Sixteen research groups contributed data from 19 samples consisting of 5199 patients, 4877 controls, and 725 FHR participants (unaffected relatives of psychosis patients), totaling 10,801 participants. Table 1 lists the data from each sample that was provided to the central site (Massachusetts General Hospital). Details for each data modality are provided in the sections below. Each research group provided the central site with detailed sample information (see Supplementary Materials), including recruitment (source, target diagnosis, illness stage [e.g. first-episode sample]), inclusion/exclusion criteria (ranges of age, IQ and years of education; substance and medication use, MRI contraindications), and data modalities, which the central site reviewed and obtained clarification as necessary. Some samples have been previously contributed to other research consortia or the data made available in repositories (see Supplementary Materials).

\subsection{Demographic and clinical characteristics of samples}

Table 2 shows the demographic and clinical characteristics of the 19 samples. The patient diagnoses consist of $76.4 \%$ schizophrenia, $8.9 \%$ schizoaffective disorder (SAD), 1.8\% schizophreniform disorder (SPD), $6.5 \%$ bipolar disorder with psychosis (BD), and 6.3\% other psychoses.

Fourteen samples consist of controls and patients with a range of illness durations, except for one sample (GAP) consists of only first-episode patients and controls. Four of these 14 samples also contain FHR individuals. Two samples consist of FHR and controls, two samples consist of only patients, and one sample consists of only controls. Given the

Table 1

Description of the GENUS Consortium Sample Collection."

\begin{tabular}{|c|c|c|c|c|c|c|c|c|c|c|c|c|c|}
\hline \multirow[t]{2}{*}{ Acronym } & \multirow[t]{2}{*}{ Sample } & \multirow[t]{2}{*}{ Site } & \multirow[t]{2}{*}{ GWAS Array } & \multicolumn{5}{|c|}{ Neuropsychological data } & \multicolumn{5}{|c|}{ T1-weighted structural MRI data ${ }^{\S}$} \\
\hline & & & & $\begin{array}{l}\text { Patients } \\
(\mathrm{N})\end{array}$ & $\begin{array}{l}\text { Controls } \\
(\mathrm{N})\end{array}$ & $\begin{array}{l}\text { FHR } \\
(\mathrm{N})\end{array}$ & $\begin{array}{l}\text { Male } \\
(\%)\end{array}$ & $\begin{array}{l}\text { Eur } \\
(\%)^{\ddagger}\end{array}$ & $\begin{array}{l}\text { Patients } \\
(\mathrm{N})\end{array}$ & $\begin{array}{l}\text { Controls } \\
(\mathrm{N})\end{array}$ & $\begin{array}{l}\text { FHR } \\
(\mathrm{N})\end{array}$ & $\begin{array}{l}\text { Male } \\
(\%)\end{array}$ & $\begin{array}{l}\text { Eur } \\
(\%)^{\ddagger}\end{array}$ \\
\hline CAMH & $\begin{array}{l}\text { Centre for Addiction and Mental } \\
\text { Health }\end{array}$ & $\begin{array}{l}\text { Toronto, } \\
\text { Canada }\end{array}$ & Illumina OmniExpress & 123 & 144 & 0 & 56.2 & 76.1 & 89 & 115 & 0 & 55.4 & 76.5 \\
\hline CATIE & $\begin{array}{l}\text { Clinical Antipsychotic Trials of } \\
\text { Intervention Effectiveness }\end{array}$ & $\begin{array}{l}\text { Multi-site, } \\
\text { USA }\end{array}$ & $\begin{array}{l}\text { Affymetrix } 500 \mathrm{~K} \text {; } \\
\text { Perlegen's custom } 164 \mathrm{~K} \\
\text { chip }\end{array}$ & 741 & 0 & 0 & 73.6 & 54.7 & - & - & - & - & - \\
\hline CIDAR/VA & $\begin{array}{l}\text { Boston Center for Intervention } \\
\text { Development and Applied } \\
\text { Research/VA Healthcare System }\end{array}$ & Boston, USA & Illumina OmniExpress & 76 & 107 & 6 & 68.8 & 60.0 & 68 & 101 & 6 & 68.0 & 59.4 \\
\hline COGS-UK & $\begin{array}{l}\text { Cognition and Genetics in } \\
\text { Schizophrenia \& Bipolar Disorder }\end{array}$ & Cardiff, UK & $\begin{array}{l}\text { Illumina Infinium } \\
\text { OmniExpressExome-8 }\end{array}$ & 835 & 0 & 0 & 58.8 & 97.3 & - & - & - & - & - \\
\hline GAP & $\begin{array}{l}\text { Genetics and Psychosis } \\
\text { First-Episode Study }\end{array}$ & London, UK & $\begin{array}{l}\text { Illumina HumanCore- } 24 \\
\text { Exome BeadChip }\end{array}$ & 164 & 160 & 0 & 59.6 & 46.8 & 132 & 94 & 0 & 56.2 & 35.0 \\
\hline IMH-SIGNRP & $\begin{array}{l}\text { Institute of Mental Health - } \\
\text { Singapore Imaging Genetics and } \\
\text { Neuropsychological Research in } \\
\text { Psychosis }\end{array}$ & Singapore & $\begin{array}{l}\text { Illumina Human } \\
\text { OmniZhongHua-8; Illumina } \\
\text { Human1M-Duo; Affymetrix } \\
6.0\end{array}$ & 150 & 63 & 0 & 55.9 & 0 & 243 & 81 & 0 & 62.4 & 0 \\
\hline IMH-STCRP & $\begin{array}{l}\text { Institute of Mental Health - } \\
\text { Singapore Translational and } \\
\text { Clinical Research in Psychosis }\end{array}$ & Singapore & $\begin{array}{l}\text { Illumina } \\
\text { HumanOmniZhongHua-8 } \\
\text { BeadChip }\end{array}$ & 420 & 1012 & 0 & 52.9 & 0 & - & - & - & - & - \\
\hline KCL-MFS & $\begin{array}{l}\text { King's College London - Maudsley } \\
\text { Family Study }\end{array}$ & London, UK & Affymetrix 6.0 & 183 & 120 & 278 & 48.0 & 95.1 & - & - & - & - & - \\
\hline KCL-MTS & $\begin{array}{l}\text { King's College London - Maudsley } \\
\text { Twin Study }\end{array}$ & London, UK & Affymetrix 6.0 & 127 & 297 & 47 & 42.9 & 100 & 63 & 75 & 23 & 60.3 & 94.5 \\
\hline$L \& R$ & $\begin{array}{l}\text { Language and Risk in } \\
\text { Schizophrenia }\end{array}$ & Boston, USA & $\begin{array}{l}\text { Illumina Infinium } \\
\text { PsychArray* }\end{array}$ & 0 & 31 & 44 & 34.7 & 74.7 & 0 & 33 & 51 & 33.3 & 71.4 \\
\hline MCIC & Mind Clinical Imaging Consortium & $\begin{array}{l}\text { Multi-site, } \\
\text { USA }\end{array}$ & $\begin{array}{l}\text { Illumina } \\
\text { HumanOmni1-Quad } \\
\text { BeadChip }\end{array}$ & 112 & 95 & 0 & 72.0 & 75.3 & 118 & 97 & 0 & 71.2 & 76.7 \\
\hline MGH & Massachusetts General Hospital & Boston, USA & $\begin{array}{l}\text { Illumina Infinium } \\
\text { PsychArray* }\end{array}$ & 434 & 0 & 0 & 72.4 & 68.8 & 61 & 123 & 0 & 65.2 & 73.2 \\
\hline NEFS & New England Family Study & Boston, USA & $\begin{array}{l}\text { Illumina Infinium } \\
\text { PsychArray* }\end{array}$ & 83 & 151 & 33 & 44.6 & 86.2 & 72 & 155 & 20 & 44.5 & 85.8 \\
\hline PAGES & Phenomics and Genomics Sample & $\begin{array}{l}\text { Munich, } \\
\text { Germany }\end{array}$ & $\begin{array}{l}\text { Illumina OmniExpress; } \\
\text { Illumina HumanHap300 }\end{array}$ & 210 & 1341 & 0 & 50.0 & 99.6 & - & - & - & - & - \\
\hline PHRS & Pittsburgh High Risk Study & $\begin{array}{l}\text { Pittsburgh, } \\
\text { USA }\end{array}$ & $\begin{array}{l}\text { Illumina Infinium } \\
\text { PsychArray* }\end{array}$ & 0 & 53 & 77 & 45.4 & 41.0 & 0 & 46 & 67 & 44.3 & 55.8 \\
\hline TCD/NUIG & $\begin{array}{l}\text { Trinity College Dublin/National } \\
\text { University of Ireland, Galway }\end{array}$ & $\begin{array}{l}\text { Multi-site, } \\
\text { Ireland }\end{array}$ & $\begin{array}{l}\text { Affymetrix 6.0; Illumina } \\
\text { HumanCore Exome }\end{array}$ & 904 & 290 & 0 & 60.9 & 99.9 & 175 & 312 & 0 & 56.9 & 99.8 \\
\hline UMCU-SZ1 & $\begin{array}{l}\text { University Medical Center Utrecht } \\
\text { - Schizophrenia Study } 1\end{array}$ & $\begin{array}{l}\text { Utrecht, } \\
\text { Netherlands }\end{array}$ & $\begin{array}{l}\text { Illumina HumanHap550; } \\
\text { Illumina Infinium } \\
\text { OmniExpressExome-8 }\end{array}$ & 97 & 143 & 0 & 68.3 & 98.6 & 159 & 157 & 0 & 69.3 & 99.1 \\
\hline UMCU-SZ2 & $\begin{array}{l}\text { University Medical Center Utrecht } \\
\text { - Schizophrenia Study } 2\end{array}$ & $\begin{array}{l}\text { Utrecht, } \\
\text { Netherlands }\end{array}$ & $\begin{array}{l}\text { Illumina HumanHap550; } \\
\text { Affymetrix 6.0; Illumina } \\
\text { Infinium } \\
\text { OmniExpressExome-8 }\end{array}$ & 233 & 144 & 235 & 58.8 & 97.5 & 184 & 131 & 212 & 59.0 & 93.6 \\
\hline $\mathrm{ZHH}$ & Zucker Hillside Hospital & $\begin{array}{l}\text { New York, } \\
\text { USA }\end{array}$ & Illumina OmniExpress & 0 & 219 & 0 & 49.3 & 100 & - & - & - & - & - \\
\hline TOTAL & & & & 4892 & 4370 & 720 & 56.5 & 72.2 & 1364 & 1520 & 379 & 57.4 & 65.0 \\
\hline
\end{tabular}

Eur = European-derived ancestry; FHR = familial high-risk.

\# Data in this table are based on the total GENUS sample collection; data for the subset with genotype data are provided in Supplementary Table 1.

$\S$ All samples with T1 MRI scans also have diffusion-weighted MRI scans except the PHRS, UMCU-SZ1, and UMCU-SZ2 samples.

$¥$ Population ancestry determined from genetic data (where available) or self-report.

* Samples genotyped at the central GENUS site. 
Table 2

Clinical and demographic characteristics of the GENUS Consortium Sample Collection. ${ }^{\#}$

\begin{tabular}{|c|c|c|c|c|c|c|c|c|c|}
\hline & \multicolumn{2}{|l|}{ Patients } & \multicolumn{2}{|l|}{ Controls } & \multicolumn{2}{|c|}{ Familial High Risk } & \multirow[t]{2}{*}{ Statistic } & \multirow[t]{2}{*}{ df } & \multirow[t]{2}{*}{$\mathrm{p}$} \\
\hline & $\mathrm{N}$ & Mean \pm SD (Range) & $\mathrm{N}$ & $\begin{array}{l}\text { Mean } \pm \text { SD } \\
\text { (Range) }\end{array}$ & $\mathrm{N}$ & $\begin{array}{l}\text { Mean } \pm \text { SD } \\
\text { (Range) }\end{array}$ & & & \\
\hline Age (years) & 5197 & $39.3 \pm 12.2(13-82)$ & 4877 & $39.2 \pm 15.8(8-86)$ & 725 & $\begin{array}{l}34.9 \pm 16.0 \\
(10-85)\end{array}$ & $\mathrm{F}=31.2$ & $\begin{array}{l}2, \\
10,796\end{array}$ & $\begin{array}{l}<1 \times \\
10^{-10}\end{array}$ \\
\hline Education Level (years) & 4697 & $12.3 \pm 2.6(1-24)$ & 4031 & $13.3 \pm 2.6(4-26)$ & 721 & $13.1 \pm 3.2(3-24)$ & $\begin{array}{l}F= \\
163.4\end{array}$ & 2, 9446 & $\begin{array}{l}<1 \times \\
10^{-10}\end{array}$ \\
\hline Premorbid IQ & 3145 & $\begin{array}{l}97.1 \pm 15.5 \\
(44-145)\end{array}$ & 1393 & $\begin{array}{l}107.6 \pm 10.7 \\
(62-145)\end{array}$ & 83 & $\begin{array}{l}105.1 \pm 16.0 \\
(45-134)\end{array}$ & $\begin{array}{l}F= \\
263.4\end{array}$ & 2,4618 & $\begin{array}{l}<1 \times \\
10^{-10}\end{array}$ \\
\hline Current IQ & 1889 & $\begin{array}{l}93.8 \pm 18.1 \\
(47-155)\end{array}$ & 2779 & $\begin{array}{l}113.4 \pm 14.9 \\
(67-161)\end{array}$ & 602 & $\begin{array}{l}105.1 \pm 15.6 \\
(58-152)\end{array}$ & $\begin{array}{l}F= \\
817.8\end{array}$ & 2,5267 & $\begin{array}{l}<1 \times \\
10^{-10}\end{array}$ \\
\hline Illness Duration (years) & 4165 & $15.1 \pm 11.6(<1-58)$ & - & - & - & - & - & - & - \\
\hline Age at Onset (years) & 4124 & $23.8 \pm 8.6(1-71)$ & - & - & - & - & - & - & - \\
\hline Global Assessment of Functioning & 1764 & $\begin{array}{l}59.8 \pm 15.9 \\
(11-100)\end{array}$ & - & - & - & - & - & - & - \\
\hline PANSS Positive symptoms & 2916 & $16.3 \pm 7.3(7-47)$ & - & - & - & - & - & - & - \\
\hline PANSS Negative Symptoms & 2912 & $16.7 \pm 7.1(7-43)$ & - & - & - & - & - & - & - \\
\hline PANSS General Symptoms & 2919 & $32.0 \pm 11.8(0-93)$ & - & - & - & - & - & - & - \\
\hline SAPS Positive Symptoms & 1533 & $7.9 \pm 12.3(0-121)$ & - & - & - & - & - & - & - \\
\hline SANS Negative Symptoms & 983 & $23.6 \pm 20.1(0-103)$ & - & - & - & - & - & - & - \\
\hline Antipsychotic dose - current CPZEQ & 3315 & $\begin{array}{l}384.2 \pm 406.6 \\
(0-5000)\end{array}$ & - & - & - & - & - & - & - \\
\hline \multirow[t]{2}{*}{$\begin{array}{l}\text { Antipsychotic dose - lifetime average } \\
\text { CPZEQ }\end{array}$} & 1433 & $\begin{array}{l}338.3 \pm 365.1 \\
(0-3125)\end{array}$ & - & - & - & - & - & - & - \\
\hline & $\mathrm{N}$ & $\%$ & $\mathrm{~N}$ & $\%$ & $\mathrm{~N}$ & $\%$ & & & \\
\hline Sex (male/female; \% male) & $3417 / 1781$ & 65.7 & $2419 / 2458$ & 49.6 & $317 / 408$ & 43.7 & $\begin{array}{l}\chi^{2}= \\
322.9\end{array}$ & 2 & $\begin{array}{l}<1 \times \\
10^{-10}\end{array}$ \\
\hline \multicolumn{10}{|l|}{ Antipsychotic medication exposure } \\
\hline Atypical & 2100 & 49.1 & - & - & - & - & - & - & - \\
\hline Typical & 411 & 9.6 & - & - & - & - & - & - & - \\
\hline Both Typical and Atypical & 544 & 12.7 & - & - & - & - & - & - & - \\
\hline Naïve/None & 474 & 11.1 & - & - & - & - & - & - & - \\
\hline Unknown Class & 324 & 7.6 & - & - & - & - & - & - & - \\
\hline No information & 422 & 9.9 & - & - & - & - & - & - & - \\
\hline \multicolumn{10}{|l|}{ Diagnosis } \\
\hline Schizophrenia & 3973 & 76.4 & - & - & - & - & - & - & - \\
\hline Schizoaffective Disorder & 465 & 8.9 & - & - & - & - & - & - & - \\
\hline Schizophreniform Disorder & 93 & 1.8 & - & - & - & - & - & - & - \\
\hline Bipolar Psychosis & 338 & 6.5 & - & - & - & - & - & - & - \\
\hline Other Psychosis & 204 & 3.9 & - & - & - & - & - & - & - \\
\hline Psychosis Unknown Type & 126 & 2.4 & - & - & - & - & - & - & - \\
\hline Ancestral Population & & & & & & & $\begin{array}{l}\chi^{2}= \\
567.6\end{array}$ & 12 & $\begin{array}{l}<1 \times \\
10^{-10}\end{array}$ \\
\hline European & 3686 & 71.2 & 3396 & 69.7 & 632 & 87.2 & - & - & - \\
\hline East Asian & 697 & 13.5 & 1117 & 22.9 & 3 & 0.4 & - & - & - \\
\hline African & 510 & 9.9 & 152 & 3.1 & 57 & 7.9 & - & - & - \\
\hline American (Predominantly Latino) & 140 & 2.7 & 30 & 0.6 & 3 & 0.4 & - & - & - \\
\hline South Asian & 50 & 1.0 & 35 & 0.7 & 7 & 1.0 & - & - & - \\
\hline Mixed & 28 & 0.5 & 11 & 0.2 & 10 & 1.4 & - & - & - \\
\hline No information & 68 & 1.3 & 135 & 2.8 & 13 & 1.8 & & & \\
\hline Handedness (right/other; \% & $2322 / 260$ & 89.9 & $2378 / 252$ & 90.4 & $609 / 59$ & 91.2 & $\chi^{2}=1.0$ & 2 & 0.60 \\
\hline
\end{tabular}

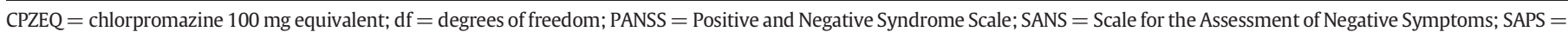
Scale for the Assessment of Positive Symptoms; SD = Standard Deviation.

\# Data in this table are based on the total GENUS sample collection; data for the subset with genotype data are provided in Supplementary Table 2.

range of illness duration ( $<1-58$ years) and the inclusion of FHR participants, the sample collection has a wide age range ( $8-86$ years). The sex composition is $56.5 \%$ male and $43.5 \%$ female. There are significant differences between the patient, control, and FHR groups in age (younger FHR), sex ratio (more male patients), years of education (fewer in patients), and ancestral population (all $p<1 \times 10^{-10}$; Table 2 ), but not in handedness. These differences must be adjusted in analyses, or matched subsets selected.

The most common clinical data across the samples are the Positive and Negative Syndrome Scale (PANSS; 54.7\% of patients) (Kay et al., 1987; Peralta and Cuesta, 1994), Scale for the Assessment of Negative Symptoms (SANS) (Andreasen, 1983) and Scale for the Assessment of Positive Symptoms (SAPS) (Andreasen, 1984) (29.5\% of patients), and Global Assessment of Functioning (GAF; 33.9\% of patients) (American Psychiatric Association, 2000).
Current or lifetime average dose of antipsychotic medication (chlorpromazine equivalents) (Gardner et al., 2010; Woods, 2003) is available for $63.8 \%$ or $27.6 \%$ of patients, respectively, and $21.2 \%$ of patients have both dosage estimates. Dosages are similar to other clinical samples (Eum et al., 2017; van Erp et al., 2016), suggesting that this patient collection is representative of and generalizable to the clinical population.

\subsection{Neuropsychological measures}

All 19 samples have neuropsychological data from 4892 patients (75.6\% schizophrenia, 9.4\% SAD, 1.7\% SPD, 6.8\% BD, 6.5\% other psychosis), 4370 controls, and 720 FHR individuals (9982 participants or $92.4 \%$ of sample; Table 1 ). The most common tests administered across the samples are shown in Table 3, with highest overlap across samples for Digit Symbol Coding, Verbal Fluency, and Word List Learning. 
Table 3

Core neuropsychological tests available for GENUS Consortium samples."

\begin{tabular}{|c|c|c|c|c|c|c|c|c|c|c|c|c|}
\hline \multirow[b]{2}{*}{ Sample } & \multicolumn{3}{|c|}{ Attention/Processing Speed } & \multicolumn{2}{|c|}{ Attention/Vigilance } & \multicolumn{2}{|c|}{ Working Memory - verbal } & \multicolumn{2}{|c|}{ Working Memory - non-verbal } & \multicolumn{3}{|c|}{ Verbal Learning \& Memory } \\
\hline & $\begin{array}{l}\text { Digit Symbol } \\
\text { Coding }\end{array}$ & TMT-A* & Verbal Fluency & CPT-IP* & Other & $\begin{array}{l}\text { Letter-Number } \\
\text { Span }\end{array}$ & Other & Spatial Span & Other & $\begin{array}{l}\text { Word List } \\
\text { Learning }\end{array}$ & Story Recall & Other \\
\hline CAMH & RBANS & $\mathrm{x}$ & RBANS Semantic/COWAT & & & UMD* $^{*}$ & RBANS Digit Span & & & RBANS & RBANS & \\
\hline CATIE & & & Category Instances/COWAT & $\mathrm{x}$ & & UMD* & & & SDRT & HVLT & & \\
\hline CIDAR-VA & BACS* & $\mathrm{x}$ & $\mathrm{MCCB}^{*}$ & $\mathrm{x}$ & АСРТ & UMD* & & WMS-III* & & HVLT-R* & WMS-III or CMS & \\
\hline COGS-UK & BACS* & $\mathrm{x}$ & MCCB $^{*}$ & $\mathrm{x}$ & & UMD* & & WMS-III* & & HVLT-R* & & \\
\hline GAP & WAIS-III & $\mathrm{x}$ & Semantic/COWAT & & & & WAIS-III Digit Span & WMS-III* & $\begin{array}{l}\text { CANTAB } \\
\text { SWM }\end{array}$ & & WMS-III & \\
\hline IMH-SIGNRP & BACS* & & $\begin{array}{l}\text { BACS Category } \\
\text { Instances/COWAT }\end{array}$ & & & & BACS Digit Sequencing & & & BACS & & \\
\hline IMH-STCRP & BACS* & & $\begin{array}{l}\text { BACS } \\
\text { Category Instances }\end{array}$ & $\mathrm{x}$ & & & BACS Digit Sequencing & & & BACS & & \\
\hline KCL-MFS & WAIS-R & $\mathrm{x}$ & & & $\begin{array}{l}\text { CANTAB } \\
\text { RVIP }\end{array}$ & & $\begin{array}{l}\text { WAIS-R/WMS-R } \\
\text { Digit Span/Arithmetic }\end{array}$ & & $\begin{array}{l}\text { CANTAB } \\
\text { SWM }\end{array}$ & & WMS-R & WMS-R VerbPA \\
\hline KCL-MTS & WAIS-III-UK & $\mathrm{x}$ & Semantic/COWAT & & $\begin{array}{l}\text { CANTAB } \\
\text { RVIP }\end{array}$ & WAIS-III-UK & $\begin{array}{l}\text { WAIS-III-UK } \\
\text { Digit Span/Arithmetic }\end{array}$ & $\begin{array}{l}\text { WMS-R-UK } \\
\text { VisMem } \\
\text { Span }\end{array}$ & $\begin{array}{l}\text { CANTAB } \\
\text { SWM }\end{array}$ & & WMS-R-UK & $\begin{array}{l}\text { WMS-R-UK } \\
\text { VerbPA }\end{array}$ \\
\hline L\&R & BACS* & $\mathrm{x}$ & $\mathrm{MCCB}^{*}$ & $\mathrm{x}$ & АСРТ & UMD* & & WMS-III* & & HVLT-R* & WMS-III & \\
\hline MCIC & & $\mathrm{x}$ & D-KEFS Semantic/Phonemic & & & WAIS-III & & & & HVLT-R* & WMS-III & \\
\hline MGH & WAIS-III & & Semantic/COWAT & $\mathrm{x}$ & & WAIS-III & $\begin{array}{l}\text { WAIS-III Digit } \\
\text { Span/Arithmetic }\end{array}$ & & & CVLT & & \\
\hline NEFS & WAIS-R & & COWAT & & ACPT & & WAIS-R Digit Span & & & CVLT or CVLT-II & $\begin{array}{l}\text { WMS-R or } \\
\text { WMS-III }\end{array}$ & \\
\hline PAGES & WAIS-R-DE & $\mathrm{x}$ & Semantic/Phonemic & & $3-7$ CPT & & $\begin{array}{l}\text { WAIS-R-DE } \\
\text { Digit Span/Arithmetic }\end{array}$ & & n-back & VLMT & WMS-R-DE & $\begin{array}{l}\text { WMS-R-DE } \\
\text { VerbPA }\end{array}$ \\
\hline PHRS & & & $\begin{array}{l}\text { MAE } \\
\text { Semantic/Phonemic }\end{array}$ & $\mathrm{x}$ & A-X CPT & & & & $\begin{array}{l}\text { Cogtest } \\
\text { SWM }\end{array}$ & Cogtest & & \\
\hline TCD/NUIG & & $\mathrm{x}$ & COWAT & $\mathrm{x}$ & 1-9 CPT & WMS-III & & & $\begin{array}{l}\text { CANTAB } \\
\text { SWM } \\
\text { /n-back }\end{array}$ & CVLT-SF & WMS-III & \\
\hline UMCU-SZ1 & & & $\begin{array}{l}\text { MAE } \\
\text { Semantic/Phonemic }\end{array}$ & & $\mathrm{H}-\mathrm{Q}$ CPT & & & & & CVLT-I-NL & & \\
\hline $\begin{array}{l}\text { UMCU-SZ2 } \\
\text { ZHH }\end{array}$ & $\begin{array}{l}\text { WAIS-III-NL } \\
\text { BACS* }\end{array}$ & $\mathrm{x}$ & MCCB* $/$ COWAT & $\mathrm{x}$ & $\mathrm{H}-\mathrm{Q}$ CPT & UMD $^{*}$ & $\begin{array}{l}\text { WAIS-III-NL Arithmetic } \\
\text { WAIS-R Digit Span }\end{array}$ & WMS-III ${ }^{*}$ & n-back & $\begin{array}{l}\text { AVLT } \\
\text { HVLT-R* }\end{array}$ & & \\
\hline N patients & 3488 & 1549 & 3956 & 2337 & 703 & 2895 & 1866 & 1097 & 1644 & 3488 & 1452 & 388 \\
\hline $\mathrm{N}$ controls & 3535 & 1116 & 2826 & 1410 & 1025 & 1080 & 3248 & 610 & 904 & 2519 & 1017 & 705 \\
\hline N FHR & 396 & 196 & 280 & 119 & 381 & 79 & 347 & 76 & 89 & 384 & 82 & 177 \\
\hline $\mathrm{N}$ total & 7419 & 2861 & 7062 & 3866 & 2109 & 4054 & 5461 & 1783 & 2637 & 6391 & 2551 & 1270 \\
\hline
\end{tabular}


Table 3 (continued)

\begin{tabular}{|c|c|c|c|c|c|c|c|c|c|}
\hline \multirow[b]{2}{*}{ Sample } & \multicolumn{2}{|c|}{ Visual Learning \& Memory } & \multicolumn{3}{|c|}{ Reasoning/Problem Solving } & \multicolumn{2}{|c|}{ Visuo-spatial Ability } & \multicolumn{2}{|l|}{ Verbal Ability } \\
\hline & BVMT-R* ${ }^{*}$ & Other & TMT-B & WCST & Other & Block Design & Other & Vocabulary & Other \\
\hline САMH & & RBANS Figure Recall & $\mathrm{x}$ & & Stroop & & RBANS JOLO/Figure Copy & & \\
\hline CATIE & & & & $64-\mathrm{C}$ & WISC-III Mazes & & & & \\
\hline CIDAR-VA & BVMT-R* & & & $64-\mathrm{C}$ & NAB Mazes* & WASI & & WASI & D-KEFS Proverbs \\
\hline COGS-UK & BVMT-R* & & & & NAB Mazes* & & & & \\
\hline GAP & & WMS-III VisRep & $\mathrm{x}$ & & CANTAB SOC & WAIS-III & WAIS-III MR & & WAIS-III INF \\
\hline IMH-SIGNRP & & & & & BACS TOL & & & & \\
\hline IMH-STCRP & & & & 64-P & BACS TOL & & WASI MR/Benton JOLO & & \\
\hline KCL-MFS & & WMS-R VisRep & $\mathrm{x}$ & & CANTAB IDED & WAIS-R & WAIS-R OA/PA/PC & WAIS-R & WAIS-R COM/INF/SIM \\
\hline KCL-MTS & & WMS-R-UK VisRep/VisPA & $\mathrm{x}$ & & CANTAB IDED & WAIS-III-UK & WAIS-III-UK OA/PA/PC & WAIS-III-UK & WAIS-III-UK COM/INF/SIM \\
\hline L\&R & BVMT-R* & & & $64-\mathrm{C}$ & NAB Mazes* & WASI & & WASI & D-KEFS Proverbs \\
\hline $\mathrm{MCIC}$ & & BVRT/WMS-III Faces & $\mathrm{x}$ & & TOL & WAIS-III & & WAIS-III & WAIS-III SIM \\
\hline MGH & & & & $128-\mathrm{C} / 64-\mathrm{C}$ & & WAIS-III & WAIS-III MR/OA/PA/PC & WAIS-III & WAIS-III COM/INF/SIM \\
\hline NEFS & & WMS-III Faces/Rey CFT Recall & & $128-\mathrm{P}$ & Stroop & WAIS-R & WAIS-R PA/Rey CFT Copy & WAIS-R & WAIS-R COM/INF; RAN \\
\hline PAGES & & WMS-R-DE FigMem/VisRep/VisPA & $\mathrm{x}$ & $128-\mathrm{C}$ & TOL-DE & WAIS-R-DE & WAIS-R-DE OA/PA/PC & WAIS-R-DE & WAIS-R-DE COM/INF/SIM \\
\hline PHRS & & CNB VOLT & & $128-\mathrm{P}$ & Cogtest Go-No-Go & & & & \\
\hline TCD/NUIG & & WMS-III Faces/CANTAB PAL & $\mathrm{x}$ & & CANTAB IDED/SART & WAIS-III-R-UK & WAIS-III-R-UK MR & WAIS-III-R-UK & WAIS-III-R-UK SIM \\
\hline UMCU-SZ1 & & & & & Stroop & WAIS-III-R-NL & WAIS-III-R-NL PA & WAIS-III-R-NL & WAIS-III-R-NL COM \\
\hline UMCU-SZ2 & & & & & NAB Mazes*/RST & WAIS-III-NL & & & WAIS-III-NL INF \\
\hline ZHH & BVMT-R* & & $\mathrm{x}$ & $128-\mathrm{P}$ & NAB Mazes*/Stroop & & & & \\
\hline N patients & 897 & 1604 & 836 & 1376 & 3555 & 2260 & 1615 & 1754 & 2048 \\
\hline $\mathrm{N}$ controls & 328 & 1628 & 1408 & 835 & 2781 & 2744 & 3131 & 2425 & 2617 \\
\hline N FHR & 48 & 317 & 145 & 134 & 350 & 522 & 33 & 285 & 567 \\
\hline $\mathrm{N}$ total & 1273 & 3549 & 2389 & 2345 & 6686 & 5526 & 4779 & 4464 & 5232 \\
\hline
\end{tabular}

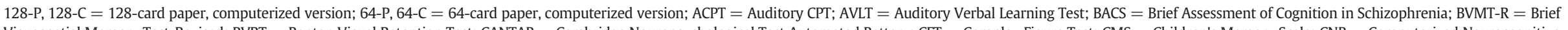

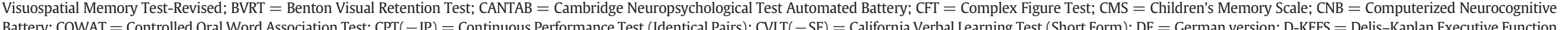

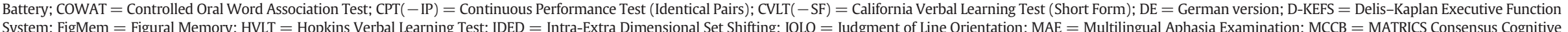

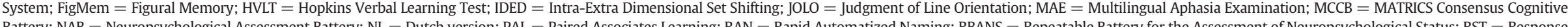

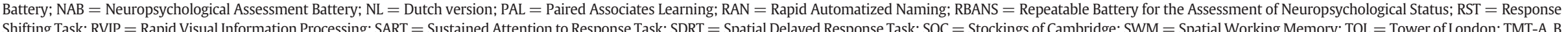

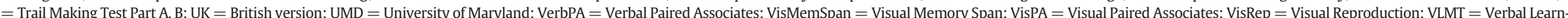

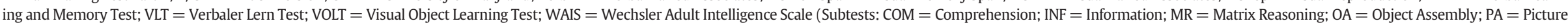
Arrangement; PC = Picture Completion; SIM = Similarities); WASI = Wechsler Abbreviated Scale of Intelligence; WCST = Wisconsin Card Sorting Test; WISC = Wechsler Intelligence Scale for Children; WMS = Wechsler Memory Scale.

$\#$ \# References for all neuropsychological tests are provided in the Supplemental Materials. Data in this table are based on the total GENUS sample collection (genotyped plus ungenotyped).

* MATRICS test. 
Supplementary Table 3 provides detailed information on the specific tests and number of participants. There are substantial differences in the mean premorbid IQ and mean current IQ between diagnostic groups (both $p<1 \times 10^{-10}$; Table 2 ). The mean premorbid and current IQ of controls and FHR individuals are higher than the population mean of 100, as previously reported by other psychosis studies (Hill et al., 2013; Seidman et al., 2015). However, the difference of $\sim 10$ IQ points between the GENUS patients and controls is consistent with the literature (Khandaker et al., 2011; Woodberry et al., 2008). Among the controls, current IQ is notably higher than premorbid IQ. The high current IQ is predominantly driven by samples that used few (2-4) WAIS subtests, which may overestimate current IQ compared to samples that used many subtests (i.e., full-scale IQ) (Axelrod, 2002). The higher current IQ may also be due to a ceiling effect, where the reading tests used to estimate premorbid IQ have a lower maximum score $(\sim 130)$ than WAIS subtests used to estimate current IQ (maximum 160).

\subsection{Neuroimaging data}

Thirteen samples have T1-weighted structural MRI scans from 1364 patients (74.4\% schizophrenia, 7.9\% SAD, 3.8\% SPD, 5.3\% BD, 8.6\% other psychosis), 1520 controls, 379 FHR individuals (3263 participants or $30 \%$ of sample; Table 1). Quality evaluation of a subset of scans from each sample discounted systematic gross errors and indicated that all datasets are high quality. In addition to the T1-weighted acquisitions, 10 samples have diffusion-weighted MRI scans from 1931 participants, and 9 samples have T2-weighted structural scans from 1821 participants. Table 4 lists the scanners and primary scan parameters for each sample. Full scan acquisition parameters are provided in the Supplementary Materials.

\subsection{SNP genotype data}

As detailed in the Supplementary Materials, 15 of the 19 samples had previously acquired raw SNP genotype data from 7478 participants (69.2\%). For 10 samples, only a proportion of participants had been genotyped. Four of the 19 samples had genomic DNA from 978 participants $(9.1 \%)$, of which 947 (8.8\%) participants had sufficient DNA quality and quantity for genotyping on the Illumina Infinium PsychArray at the central site. Table 1 lists the SNP arrays used for each sample. Supplementary Table 1 lists the number of genotyped participants in each sample and Supplementary Table 2 provides the demographic and clinical characteristics. Of the total 8425 participants with genotype data, 164 participants were excluded during quality control analyses due to low (<98\%) genotype call rate, resulting in 8261 participants with genotype data suitable for imputation (4099 patients, 3851 controls, 306 FHR). Further quality control and imputation procedures will be described elsewhere. The mean call rate across the cleaned dataset is $99.8 \%$ (range $99.3 \%-99.9 \%$ ). The sample collection has $80 \%$ power to detect a genetic variant that explains $0.5 \%$ of the variance of a phenotype at a genome-wide significant alpha $=5 \times 10^{-8}$.

The ancestry breakdown based on genotype data is 70.2\% European (2835 patients, 2703 controls, 264 FHR), 19.5\% East Asian (624 patients, 982 controls, 1 FHR), 7.3\% African (454 patients, 111 controls, 35 FHR), $2.0 \%$ American (predominantly Latino; 138 patients, 28 controls, 3 FHR), and $1.0 \%$ other ancestry (53 patients, 27 controls, 3 FHR).

\section{Discussion}

This article provides a general description of the GENUS Consortium and its sample collection, which is the largest known dataset of psychosis patients, controls, and FHR individuals with data spanning genetics, clinical, cognitive and, for a subset, structural MRI and diffusion imaging.

Table 4

MRI scan parameters for GENUS Consortium samples.

\begin{tabular}{|c|c|c|c|c|c|c|c|c|}
\hline Sample & $\begin{array}{l}\text { Magnetic Field } \\
\text { Strength }\end{array}$ & Vendor & Model & $\begin{array}{l}\text { T1-weighted } \\
\text { sequence }\end{array}$ & $\begin{array}{l}\text { T1 Voxel } \\
\text { dimensions (mm) }\end{array}$ & $\begin{array}{l}\text { DW-MRI \# } \\
\text { diffusion-encoding } \\
\text { directions }\end{array}$ & $\begin{array}{l}\text { DW-MRI b-value } \\
\left(\mathrm{s} / \mathrm{mm}^{2}\right)\end{array}$ & $\begin{array}{l}\text { DW-MRI Voxel } \\
\text { dimensions (mm) }\end{array}$ \\
\hline CAMH & $1.5 \mathrm{~T}$ & GE & Echospeed & IR-SPGR & $0.78 \times 0.78 \times 1.5$ & 23 & 1000 & $2.6 \times 2.6 \times 2.6$ \\
\hline \multirow[t]{2}{*}{ CIDAR-VA } & $3 \mathrm{~T}$ & GE & $\begin{array}{l}\text { Signa HDxt } \\
\text { Echospeed }\end{array}$ & IR-SPGR & $1.0 \times 1.0 \times 1.0$ & 51 & 900 & $1.67 \times 1.67 \times 1.7$ \\
\hline & $3 \mathrm{~T}$ & Siemens & Trio Tim & MP-RAGE & $1.0 \times 1.0 \times 1.33$ & 60 & 700 & $2.0 \times 2.0 \times 2.0$ \\
\hline GAP & $3 \mathrm{~T}$ & GE & Signa HDx & MP-RAGE & $1.01 \times 1.01 \times 1.2$ & 32 & 1300 & $2.4 \times 2.4 \times 2.4$ \\
\hline IMH-SIGNRP & $3 \mathrm{~T}$ & Philips & Intera Achieva & TFE & $0.9 \times 0.9 \times 0.9$ & 15 & 800 & $0.9 \times 0.9 \times 3.0$ \\
\hline \multirow[t]{2}{*}{ KCL-MTS } & $1.5 \mathrm{~T}$ & GE & $\begin{array}{l}\text { Signa } \\
\text { Advantage }\end{array}$ & SPGR & $0.78 \times 0.78 \times 1.5$ & 64 & 1300 & $2.5 \times 2.5 \times 2.5$ \\
\hline & $1.5 \mathrm{~T}$ & GE & $\begin{array}{l}\text { Signa } \\
\text { Advantage }\end{array}$ & SPGR & $0.78 \times 0.78 \times 1.5$ & 64 & 1300 & $2.5 \times 2.5 \times 2.5$ \\
\hline L\&R & $3 \mathrm{~T}$ & Siemens & Trio Tim & MP-RAGE & $1.0 \times 1.0 \times 1.0$ & 60 & 700 & $2.0 \times 2.0 \times 2.0$ \\
\hline \multirow[t]{3}{*}{ MCIC } & $1.5 \mathrm{~T}$ & Siemens & Sonata & GRE & $0.7 \times 0.7 \times 1.5$ & 60 & 700 & $2.0 \times 2.0 \times 2.0$ \\
\hline & $3 \mathrm{~T}$ & Siemens & Trio Tim & MP-RAGE & $\begin{array}{l}0.625 \times 0.625 \times \\
1.5\end{array}$ & 12 & 1000 & $2.0 \times 2.0 \times 2.0$ \\
\hline & $1.5 \mathrm{~T}$ & Siemens & Sonata & GRE & $\begin{array}{l}0.625 \times 0.625 \times \\
1.5\end{array}$ & 12 & 1000 & $2.0 \times 2.0 \times 2.0$ \\
\hline \multirow[t]{2}{*}{ MGH } & $3 \mathrm{~T}$ & Siemens & Trio Tim & ME-MP-RAGE & $1.2 \times 1.2 \times 1.2$ & 6 & 1000 & $1.375 \times 1.375 \times 3.0$ \\
\hline & $3 \mathrm{~T}$ & Siemens & Trio Tim & MP-RAGE & $1 \times 1 \times 1.3$ & - & - & - \\
\hline \multirow[t]{5}{*}{ NEFS } & $1.5 \mathrm{~T}$ & Siemens & Avanto & MP-RAGE & $1.0 \times 1.0 \times 1.33$ & 60 & 700 & $2.0 \times 2.0 \times 2.0$ \\
\hline & $1.5 \mathrm{~T}$ & Siemens & Sonata & MP-RAGE & $1.0 \times 1.0 \times 1.33$ & 6 & 600 & $2.0 \times 2.0 \times 2.0$ \\
\hline & $1.5 \mathrm{~T}$ & Siemens & Sonata & MP-RAGE & $1.0 \times 1.0 \times 1.5$ & - & - & - \\
\hline & $3 \mathrm{~T}$ & Siemens & Trio Tim & MP-RAGE & $1.0 \times 1.0 \times 1.33$ & 60 & 700 & $2.0 \times 2.0 \times 2.0$ \\
\hline & $1.5 \mathrm{~T}$ & GE & Genesis Signa & EFGRE & $0.94 \times 0.94 \times 1.5$ & - & - & - \\
\hline PHRS & $1.5 \mathrm{~T}$ & GE & Genesis Signa & SPGR & $1.25 \times 1.25 \times 1.5$ & - & - & - \\
\hline \multirow[t]{2}{*}{ TCD/NUIG } & $3 \mathrm{~T}$ & Philips & Intera Achieva & TFE & $0.9 \times 0.9 \times 0.9$ & 15 & 800 & $1.75 \times 1.75 \times 2.2$ \\
\hline & $1.5 \mathrm{~T}$ & Siemens & $\begin{array}{l}\text { Magnetom } \\
\text { Symphony }\end{array}$ & MP-RAGE & $0.45 \times 0.45 \times 0.9$ & - & - & - \\
\hline UMCU-SZ1 & $1.5 \mathrm{~T}$ & Philips & NT Intera & FFE & $1.0 \times 1.0 \times 1.2$ & - & - & - \\
\hline UMCU-SZ2 & $1.5 \mathrm{~T}$ & Philips & Achieva & FFE & $1.0 \times 1.0 \times 1.2$ & - & - & - \\
\hline
\end{tabular}

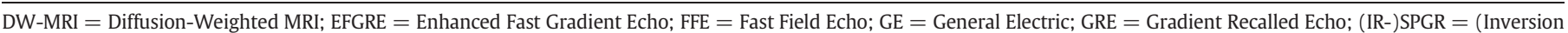
Recovery) Spoiled Gradient Recalled; (ME-)MP-RAGE = (Multi-Echo) Magnetization Prepared Rapid Acquisition Gradient Echo; TFE = Turbo Field Echo. 
This dataset enables large-scale investigations of brain-based phenotypes. Due to data sharing restrictions of many of the individual samples, the full dataset is currently only available to external researchers through collaboration with GENUS Consortium members. The extent of data and large size of the GENUS dataset, as well as the breadth of expertise of the GENUS Consortium members, provide a host of opportunities for analyses. For example, examining sex differences in diseaserelated phenotypes is an important but often overlooked aspect of psychiatric studies (Goldstein et al., 2013) that can be addressed with this large, well-phenotyped sample collection.

The GENUS Consortium differs in several aspects from other largescale efforts investigating the genetic architecture of cognition and neuroanatomy relevant to psychosis (e.g., COGENT, ENIGMA, B-SNIP, Brain Genomics Superstruct Project, Philadelphia Neurodevelopmental Cohort) (Franke et al., 2016; Germine et al., 2016; Holmes et al., 2015; Lee et al., 2016; Lencz et al., 2014; Tamminga et al., 2013). A key difference is that many other studies do not have data for both cognition and brain structure modalities from the same participants. Bridging multiple brain phenotype modalities, as in the GENUS sample collection, is important for heterogeneous disorders such as schizophrenia that are defined by diverse symptoms and abnormalities whose relationships are mostly unknown. Another difference is the GENUS subject-level data are stored at the central site, allowing for stringent quality control and site comparability analyses, and the option for mega-analyses across the entire dataset, whereas some other studies are limited to meta-analysis of results generated by each site separately.

A major strength of the GENUS sample collection is the existence of extensive data across patients, controls, and FHR individuals that enable analyses of genetic effects in multiple diagnostic groups. While informative genetic findings are emerging from large healthy cohorts, this is currently lacking in psychosis cohorts, and it remains unclear whether genetic factors influencing brain structure and function in healthy cohorts have the same effect in psychiatric patients. The GENUS Consortium analyses will initially focus on relating schizophrenia genetic risk variants identified by prior GWAS with the cognitive and brain structural phenotypes available in this sample collection. While the ENIGMA Consortium did not detect significant effects of schizophrenia genetic risk variants on subcortical volumes in mixed diagnosis and healthy individuals (Franke et al., 2016), a study of cortical thickness and surface area reported that a substantial proportion (30-45\%) of the heritability is explained by schizophrenia genetic risk variants (Lee et al., 2016). This suggests that some brain structural measures may be more genetically related to schizophrenia than others, or that genetic relationships differ in diseased and healthy brain. In addition, GWAS of cognitive performance and brain regional volumes have detected novel genetic associations (Adams et al., 2016; Davies et al., 2015; Hibar et al., 2015; Trampush et al., 2017) that could be further investigated in the GENUS sample collection.

Regarding genetic analyses, the GENUS sample collection is best suited for characterizing SNPs, polygenic factors, and pathways identified by GWAS, such as the PGC GWAS mega-analyses (PGC Schizophrenia Working Group, 2014), for effects on brain-based phenotypes, or replicating findings from other genetic studies of cognition or brain structure. Due to the small effect sizes of common genetic variants, our dataset is not well powered for GWAS discovery. SNP-based heritability approaches (e.g., GCTA) require approximately 4000 subjects for $80 \%$ power to estimate heritability as low as 20\% (Visscher et al., 2014), a reasonable assumption for cognitive and brain volume traits (Franke et al., 2016; Trampush et al., 2017); therefore, some of our phenotypes (e.g. letternumber span tests, WAIS Digit Symbol Coding) are suitable for this approach. Rare variant association studies require enormous samples for adequate statistical power (Auer and Lettre, 2015; Zuk et al., 2014), therefore our dataset is not sufficient on its own for such analyses. The availability of multiple phenotypes enables a breadth of analyses, with the caveat that significance thresholds must be adjusted for multiple testing, although accounting for correlations between phenotypes and other data reduction methods could allow for more lenient thresholds. The statistical power of our dataset could also be maximized by merging phenotypes into one phenotype, such as Spearman's ' $g$ ', in which data from many neuropsychological tests are used to derive a single measure of general cognitive ability (Spearman, 1904).

There are considerable challenges to combining data acquired by many research groups. The heterogeneity in the data collected and the protocols used by each group requires careful harmonization of the data to maximize comparability between the samples and minimize confounds. Our harmonization approaches will be described in greater detail in subsequent data-based articles. Briefly, we are applying methods that use controls from each sample to standardize the data (i.e., generate $\mathrm{Z}$ scores), as has been reported for neuropsychological data (Toulopoulou et al., 2010) and structural MRI data (Segall et al., 2009; Wilke et al., 2014). Further, variability in multi-site imaging data due to different scanner models and field strengths, acquisition protocols, and image segmentation methods (Han et al., 2006) can be minimized by processing all scans using a consistent segmentation routine, which enables detection of subtle effects (Fennema-Notestine et al., 2007), including gray matter loss in schizophrenia datasets (Segall et al., 2009). Regarding clinical data, positive and negative symptom data can be converted between the PANSS and SANS/SAPS, the most common clinical scales in our dataset, using regression-based equations (van Erp et al., 2014). As for the limited medication dosage information of our dataset, this can be addressed partially by confirming findings from the full cohort in the subset with medication data to rule out medication confounds. We are harmonizing the genotype data from various SNP arrays by imputing genotypes based on a reference panel to generate a common set of SNPs across all samples, an accepted approach in the field (PGC Schizophrenia Working Group, 2014). Although heterogeneous data collected by multiple sites is not ideal, the large volume of available legacy data with deep phenotypic and genotype information warrants maximizing its use by generating one merged dataset that has far greater statistical power than the individual samples.

In summary, the GENUS Consortium sample collection is a valuable resource that builds upon previous efforts by individual research groups and complements other psychosis datasets. This high-powered sample collection integrates measures of brain structure, cognition, and genetics for studying the biological basis of psychosis through original analyses and collaborative replication studies. There will be the opportunity for multiple publications from these data, including articles focusing on harmonization and genetic analyses of the cognitive data and imaging data, and publications that incorporate multi-modal data. The rich phenotypic data are expected to provide new insights into neural functions that are disrupted in psychosis.

\section{Contributors}

Dr. Blokland, Dr. del Re, and Dr. Petryshen drafted the manuscript. Dr. Blokland performed the statistical analyses. Dr. Petryshen designed the collaborative project. Al other authors participated in aspects of the study design (both within and across sites), including subject recruitment and data collection. All authors were responsible for reviewing, editing, and approving the final version of the manuscript.

\section{Role of funding source}

The sponsor (the National Institutes of Health) had no role in the design and conduct of the study; collection, analysis, and interpretation of data by the GENUS Consortium; and preparation, review, or approval of the manuscript. The content of this article is solely the responsibility of the authors and does not necessarily represent the official views of the National Institutes of Health.

\section{Conflict of interest}

All authors declare that they have no conflicts of interest with respect to this study.

\section{Acknowledgements}

We are grateful for the support of all study staff and participants. Acknowledgements for each sample are provided in the Supplementary Materials. Data processing and analyses (of the legacy data) at the central site was supported by the National Institute of Mental Health (NIMH) of the National Institutes of Health (NIH) grant number R01MH092380 to T.L.P. supporting the Genetics of Endophenotypes of Neurofunction to Understand Schizophrenia (GENUS) Consortium, and NIMH grant R21MH109819 to E.D.R. 


\section{Appendix A. Supplementary data}

Supplementary data to this article can be found online at https://doi. org/10.1016/j.schres.2017.09.024.

\section{References}

Adams, H.H., Hibar, D.P., Chouraki, V., Stein, J.L., Nyquist, P.A., Renteria, M.E., Trompet, S., Arias-Vasquez, A., Seshadri, S., Desrivieres, S., Beecham, A.H., Jahanshad, N., Wittfeld, K., Van der Lee, S.J., Abramovic, L., Alhusaini, S., Amin, N., Andersson, M., Arfanakis, K., Aribisala, B.S., Armstrong, N.J., Athanasiu, L., Axelsson, T., Beiser, A., Bernard, M., Bis, J.C., Blanken, L.M., Blanton, S.H., Bohlken, M.M., Boks, M.P., Bralten, J., Brickman, A.M., Carmichael, O., Chakravarty, M.M., Chauhan, G., Chen, Q., Ching, C.R., Cuellar-Partida, G., Braber, A.D., Doan, N.T., Ehrlich, S., Filippi, I., Ge, T., Giddaluru, S., Goldman, A.L., Gottesman, R.F., Greven, C.U., Grimm, O., Griswold M.E., Guadalupe, T., Hass, J., Haukvik, U.K., Hilal, S., Hofer, E., Hoehn, D., Holmes, A.J., Hoogman, M., Janowitz, D. Jia, T. Kasperaviciute, D., Kim, S., Klein, M., Kraemer, B. Lee, P.H., Liao, J., Liewald, D.C., Lopez, L.M., Luciano, M., Macare, C., Marquand, A, Matarin, M., Mather, K.A., Mattheisen, M., Mazoyer, B., McKay, D.R., McWhirter, R., Milaneschi, Y., Mirza-Schreiber, N., Muetzel, R.L., Maniega, S.M., Nho, K., Nugent, A.C., Loohuis, L.M., Oosterlaan, J., Papmeyer, M., Pappa, I., Pirpamer, L., Pudas, S., Putz, B., Rajan, K.B., Ramasamy, A., Richards, J.S., Risacher, S.L., Roiz-Santianez, R., Rommelse, N., Rose, E.J., Royle, N.A., Rundek, T., Samann, P.G., Satizabal, C.L. Schmaal, L., Schork, A.J., Shen, L., Shin, J., Shumskaya, E., Smith, A.V., Sprooten, E. Strike, L.T., Teumer, A., Thomson, R., Tordesillas-Gutierrez, D., Toro, R., Trabzuni, D., Vaidya, D., Van der Grond, J., Van der Meer, D., Van Donkelaar, M.M., Van Eijk, K.R., Van Erp, T.G., Van Rooij, D., Walton, E., Westlye, L.T., Whelan, C.D., Windham, B.G., Winkler, A.M., Woldehawariat, G., Wolf, C., Wolfers, T., Xu, B., Yanek, L.R., Yang, J. Zijdenbos, A., Zwiers, M.P., Agartz, I., Aggarwal, N.T., Almasy, L., Ames, D., Amouyel, P., Andreassen, O.A., Arepalli, S., Assareh, A.A., Barral, S., Bastin, M.E., Becker, D.M. Becker, J.T., Bennett, D.A., Blangero, J., van Bokhoven, H., Boomsma, D.I., Brodaty, H., Brouwer, R.M., Brunner, H.G., Buckner, R.L., Buitelaar, J.K., Bulayeva, K.B., Cahn, W., Calhoun, V.D., Cannon, D.M., Cavalleri, G.L., Chen, C., Cheng, C.Y., Cichon, S. Cookson, M.R., Corvin, A., Crespo-Facorro, B., Curran, J.E., Czisch, M., Dale, A.M., Davies, G.E., De Geus, E.J., De Jager, P.L., de Zubicaray, G.I., Delanty, N., Depondt, C. DeStefano, A.L., Dillman, A., Djurovic, S., Donohoe, G., Drevets, W.C., Duggirala, R., Dyer, T.D., Erk, S., Espeseth, T., Evans, D.A., Fedko, I.O., Fernandez, G., Ferrucci, L., Fisher, S.E. Fleischman, D.A, Ford, I., Foroud, T.M., Fox, P.T, Francks, C, Fukunaga M., Gibbs, J.R., Glahn, D.C., Gollub, R.L., Goring, H.H., Grabe, H.J., Green, R.C., Gruber, O., Gudnason, V., Guelfi, S., Hansell, N.K., Hardy, J., Hartman, C.A., Hashimoto, R. Hegenscheid, K., Heinz, A., Le Hellard, S., Hernandez, D.G., Heslenfeld, D.J., Ho, B.C. Hoekstra, P.J., Hoffmann, W., Hofman, A., Holsboer, F., Homuth, G., Hosten, N. Hottenga, J.J., Pol, H.E., Ikeda, M., Ikram, M.K., Jack Jr., C.R., Jenkinson, M., Johnson, R., Jonsson, E.G., Jukema, J.W., Kahn, R.S., Kanai, R., Kloszewska, I., Knopman, D.S., Kochunov, P., Kwok, J.B., Lawrie, S.M., Lemaitre, H., Liu, X., Longo, D.L., Longstreth Jr., W.T., Lopez, O.L., Lovestone, S., Martinez, O., Martinot, J.L., Mattay, V.S. McDonald, C., McIntosh, A.M., McMahon, K.L., McMahon, F.J., Mecocci, P., Melle, I. Meyer-Lindenberg, A., Mohnke, S., Montgomery, G.W., Morris, D.W., Mosley, T.H. Muhleisen, T.W., Muller-Myhsok, B., Nalls, M.A., Nauck, M., Nichols, T.E., Niessen, W.J., Nothen, M.M., Nyberg, L., Ohi, K., Olvera, R.L., Ophoff, R.A., Pandolfo, M., Paus, T., Pausova, Z., Penninx, B.W., Pike, G.B., Potkin, S.G., Psaty, B.M., Reppermund, S. Rietschel, M., Roffman, J.L., Romanczuk-Seiferth, N., Rotter, J.I., Ryten, M., Sacco, R.L., Sachdev, P.S., Saykin, A.J., Schmidt, R., Schofield, P.R., Sigurdsson, S., Simmons, A., Singleton, A., Sisodiya, S.M., Smith, C., Smoller, J.W., Soininen, H., Srikanth, V., Steen, V.M., Stott, D.J., Sussmann, J.E., Thalamuthu, A., Tiemeier, H., Toga, A.W., Traynor, B.J., Troncoso, J., Turner, J.A., Tzourio, C., Uitterlinden, A.G., Hernandez, M.C., Van der Brug, M., Van der Lugt, A., Van der Wee, N.J., Van Duijn, C.M., Van Haren, N.E., Van, T.E.D., Van Tol, M.J., Vardarajan, B.N., Veltman, D.J., Vernooij, M.W., Volzke, H. Walter, H., Wardlaw, J.M., Wassink, T.H., Weale, M.E., Weinberger, D.R., Weiner, M.W., Wen, W., Westman, E., White, T., Wong, T.Y., Wright, C.B., Zielke, H.R. Zonderman, A.B., Deary, I.J., DeCarli, C., Schmidt, H., Martin, N.G., De Craen, A.J. Wright, M.J., Launer, L.J., Schumann, G., Fornage, M., Franke, B., Debette, S., Medland, S.E., Ikram, M.A., Thompson, P.M., 2016. Novel genetic loci underlying human intracranial volume identified through genome-wide association. Nat. Neurosci. 19 (12), 1569-1582.

American Psychiatric Association, 2000. Diagnostic and Statistical Manual of Mental Disorders, Fourth Edition, Text Revision (DSM-IV-TR). American Psychiatric Press Inc. Washington, DC

Andreasen, N.C., 1983. Scale for the Assessment of Negative Symptoms (SANS). University of Iowa, Iowa City.

Andreasen, N.C., 1984. Scale for the Assessment of Positive Symptoms (SAPS). University of Iowa, Iowa City.

Auer, P.L., Lettre, G., 2015. Rare variant association studies: Considerations, challenges and opportunities. Genome Med. 7 (1), 16

Axelrod, B.N., 2002. Validity of the Wechsler Abbreviated Scale of Intelligence and other very short forms of estimating intellectual functioning. Assessment 9 (1), 17-23.

Blokland, G.A.M., de Zubicaray, G.I., McMahon, K.L., Wright, M.J., 2012. Genetic and environmental influences on neuroimaging phenotypes: A meta-analytical perspective on twin imaging studies. Twin Res. Hum. Genet. Off. J. Int. Soc. Twin Stud. 15 (3), 351-371.

Blokland, G.A.M., Mesholam-Gately, R.I., Toulopoulou, T., del Re, E.C., Lam, M., DeLisi, L.E., Donohoe, G., Walters, J.T.R., GENUS Consortium, Seidman, L.J., Petryshen, T.P., 2017. Heritability of neuropsychological measures in schizophrenia and non-psychiatric populations: A systematic review and meta-analysis. Schizophr. Bull. 43 (4), 788-800.
Bohlken, M.M., Brouwer, R.M., Mandl, R.C., Kahn, R.S., Hulshoff Pol, H.E., 2016. Genetic variation in schizophrenia liability is shared with intellectual ability and brain structure. Schizophr. Bull. 42 (5), 1167-1175

Boos, H.B., Aleman, A., Cahn, W., Hulshoff Pol, H.E., Kahn, R.S., 2007. Brain volumes in relatives of patients with schizophrenia: A meta-analysis. Arch. Gen. Psychiatry 64 (3), 297-304.

Bora, E., Fornito, A., Radua, J., Walterfang, M., Seal, M., Wood, S.J., Yucel, M., Velakoulis, D., Pantelis, C., 2011. Neuroanatomical abnormalities in schizophrenia: A multimodal voxelwise meta-analysis and meta-regression analysis. Schizophr. Res. 127 (1-3), 46-57.

CNV and Schizophrenia Working Groups of the Psychiatric Genomics Consortium; Psychosis Endophenotypes International Consortium, 2017. Contribution of copy number variants to schizophrenia from a genome-wide study of 41,321 subjects. Nat. Genet. 49 (1), 27-35.

Davies, G, Armstrong N., Bis, J.C. Bressler, J., Chouraki, V., Giddaluru, S, Hofer, E. IbrahimVerbaas, C.A., Kirin, M., Lahti, J., van der Lee, S.J., Le Hellard, S., Liu, T., Marioni, R.E., Oldmeadow, C., Postmus, I., Smith, A.V., Smith, J.A., Thalamuthu, A., Thomson, R., Vitart, V., Wang, J., Yu, L., Zgaga, L., Zhao, W., Boxall, R., Harris, S.E., Hill, W.D., Liewald, D.C., Luciano, M., Adams, H., Ames, D., Amin, N., Amouyel, P., Assareh, A.A., $\mathrm{Au}$, R., Becker, J.T., Beiser, A., Berr, C., Bertram, L., Boerwinkle, E., Buckley, B.M., Campbell, H., Corley, J., De Jager, P.L., Dufouil, C., Eriksson, J.G., Espeseth, T., Faul, J.D., Ford, I., Generation, S., Gottesman, R.F., Griswold, M.E., Gudnason, V., Harris, T.B., Heiss, G., Hofman, A., Holliday, E.G., Huffman, J., Kardia, S.L., Kochan, N., Knopman, D.S., Kwok, J.B., Lambert, J.C., Lee, T., Li, G., Li, S.C., Loitfelder, M., Lopez, O.L., Lundervold, A.J., Lundqvist, A., Mather, K.A., Mirza, S.S., Nyberg, L., Oostra, B.A., Palotie, A., Papenberg, G., Pattie, A., Petrovic, K., Polasek, O., Psaty, B.M., Redmond, P., Reppermund, S., Rotter, J.I., Schmidt, H., Schuur, M., Schofield, P.W., Scott, R.J., Steen, V.M., Stott, D.J., van Swieten, J.C., Taylor, K.D., Trollor, J., Trompet, S., Uitterlinden, A.G., Weinstein, G., Widen, E., Windham, B.G., Jukema, J.W., Wright, A.F., Wright, M.J., Yang, Q., Amieva, H., Attia, J.R., Bennett, D.A., Brodaty, H., de Craen, A.J., Hayward, C., Ikram, M.A., Lindenberger, U., Nilsson, L.G., Porteous, D.J., Raikkonen, K., Reinvang, I., Rudan, I., Sachdev, P.S., Schmidt, R., Schofield, P.R., Srikanth, V., Starr, J.M., Turner, S.T., Weir, D.R., Wilson, J.F., van Duijn, C., Launer, L., Fitzpatrick, A.L., Seshadri, S., Mosley Jr., T.H., Deary, I.J., 2015. Genetic contributions to variation in general cognitive function: A meta-analysis of genome-wide association studies in the CHARGE consortium $(\mathrm{N}=53949)$. Mol. Psychiatry 20 (2), 183-192.

Donohoe, G., Morris, D.W., Corvin, A., 2010. The psychosis susceptibility gene ZNF804A: Associations, functions, and phenotypes. Schizophr. Bull. 36 (5), 904-909.

Donohoe, G., Walters, J., Hargreaves, A., Rose, E.J., Morris, D.W., Fahey, C., Bellini, S. Cummins, E., Giegling, I., Hartmann, A.M., Moller, H.J., Muglia, P., Owen, M.J., Gill, M., O'Donovan, M.C., Tropea, D., Rujescu, D., Corvin, A., 2013. Neuropsychological effects of the CSMD1 genome-wide associated schizophrenia risk variant rs10503253. Genes Brain Behav. 12 (2), 203-209.

Eum, S., Hill, S.K., Rubin, L.H., Carnahan, R.M., Reilly, J.L., Ivleva, E.I., Keedy, S.K., Tamminga, C.A., Pearlson, G.D., Clementz, B.A., Gershon, E.S., Keshavan, M.S., Keefe, R.S., Sweeney, J.A., Bishop, J.R., 2017. Cognitive burden of anticholinergic medications in psychotic disorders. Schizophr. Res. https://doi.org/10.1016/j.schres.2017.03.034 (Epub ahead of print).

Fedorov, A., Beichel, R., Kalpathy-Cramer, J., Finet, J., Fillion-Robin, J.C., Pujol, S., Bauer, C., Jennings, D., Fennessy, F., Sonka, M., Buatti, J., Aylward, S., Miller, J.V., Pieper, S., Kikinis, R., 2012. 3D Slicer as an image computing platform for the Quantitative Imaging Network. Magn. Reson. Imaging 30 (9), 1323-1341.

Fennema-Notestine, C., Gamst, A.C., Quinn, B.T., Pacheco, J., Jernigan, T.L., Thal, L., Buckner, R., Killiany, R., Blacker, D., Dale, A.M., Fischl, B., Dickerson, B., Gollub, R.L., 2007. Feasibility of multi-site clinical structural neuroimaging studies of aging using legacy data. Neuroinformatics 5 (4), 235-245.

Franke, B., Stein, J.L., Ripke, S., Anttila, V., Hibar, D.P., van Hulzen, K.J., Arias-Vasquez, A., Smoller, J.W., Nichols, T.E., Neale, M.C., McIntosh, A.M., Lee, P., McMahon, F.J., Meyer-Lindenberg, A., Mattheisen, M., Andreassen, O.A., Gruber, O., Sachdev, P.S., Roiz-Santianez, R., Saykin, A.J., Ehrlich, S., Mather, K.A., Turner, J.A., Schwarz, E., Thalamuthu, A., Yao, Y., Ho, Y.Y., Martin, N.G., Wright, M.J., Schizophrenia Working Group of the Psychiatric Genomics Consortium, Psychosis Endophenotypes International Consortium, Wellcome Trust Case Control Consortium, ENIGMA Consortium, O'Donovan, M.C., Thompson, P.M., Neale, B.M., Medland, S.E., Sullivan, P.F., 2016. Genetic influences on schizophrenia and subcortical brain volumes: Large-scale proof of concept. Nat. Neurosci. 19 (3), 420-431.

Gardner, D.M., Murphy, A.L., O'Donnell, H., Centorrino, F., Baldessarini, R.J., 2010. International consensus study of antipsychotic dosing. Am. J. Psychiatry 167 (6), 686-693.

Ge, T., Nichols, T.E., Lee, P.H., Holmes, A.J., Roffman, J.L., Buckner, R.L., Sabuncu, M.R., Smoller, J.W., 2015. Massively expedited genome-wide heritability analysis (MEGHA). Proc. Natl. Acad. Sci. U. S. A. 112 (8), 2479-2484.

Germine, L., Robinson, E.B., Smoller, J.W., Calkins, M.E., Moore, T.M., Hakonarson, H., Daly, M.J., Lee, P.H., Holmes, A.J., Buckner, R.L., Gur, R.C., Gur, R.E., 2016. Association between polygenic risk for schizophrenia, neurocognition and social cognition across development. Transl. Psychiatry 6 (10), e924.

Goldstein, J.M., Cherkerzian, S., Tsuang, M.T., Petryshen, T.L., 2013. Sex differences in the genetic risk for schizophrenia: History of the evidence for sex-specific and sex-dependent effects. Am. J. Med. Genet. B Neuropsychiatr. Genet. 162B (7), 698-710.

Gottesman, I.I., Gould, T.D., 2003. The endophenotype concept in psychiatry: Etymology and strategic intentions. Am. J. Psychiatry 160 (4), 636-645.

Haijma, S.V., Van Haren, N., Cahn, W., Koolschijn, P.C., Hulshoff Pol, H.E., Kahn, R.S., 2013. Brain volumes in schizophrenia: A meta-analysis in over 18,000 subjects. Schizophr. Bull. 39 (5), 1129-1138.

Han, X., Jovicich, J., Salat, D., van der Kouwe, A., Quinn, B., Czanner, S., Busa, E., Pacheco, J., Albert, M., Killiany, R., Maguire, P., Rosas, D., Makris, N., Dale, A., Dickerson, B., Fischl, 
B., 2006. Reliability of MRI-derived measurements of human cerebral cortical thickness: The effects of field strength, scanner upgrade and manufacturer. Neurolmage 32 (1), 180-194.

Hatzimanolis, A., Bhatnagar, P., Moes, A., Wang, R., Roussos, P., Bitsios, P., Stefanis, C.N., Pulver, A.E., Arking, D.E., Smyrnis, N., Stefanis, N.C., Avramopoulos, D., 2015. Common genetic variation and schizophrenia polygenic risk influence neurocognitive performance in young adulthood. Am. J. Med. Genet. B Neuropsychiatr. Genet. 168B (5), 392-401.

Hibar, D.P., Stein, J.L., Renteria, M.E., Arias-Vasquez, A., Desrivieres, S., Jahanshad, N., Toro, R., Wittfeld, K., Abramovic, L., Andersson, M., Aribisala, B.S., Armstrong, N.J., Bernard, M., Bohlken, M.M., Boks, M.P., Bralten, J., Brown, A.A., Chakravarty, M.M., Chen, Q., Ching, C.R., Cuellar-Partida, G., den Braber, A., Giddaluru, S., Goldman, A.L., Grimm, O., Guadalupe, T., Hass, J., Woldehawariat, G., Holmes, A.J., Hoogman, M., Janowitz, D., Jia, T., Kim, S., Klein, M., Kraemer, B., Lee, P.H., Olde Loohuis, L.M., Luciano, M., Macare, C., Mather, K.A., Mattheisen, M., Milaneschi, Y., Nho, K., Papmeyer, M., Ramasamy, A., Risacher, S.L., Roiz-Santianez, R., Rose, E.J., Salami, A., Samann, P.G., Schmaal, L., Schork, A.J., Shin, J., Strike, L.T., Teumer, A., van Donkelaar, M.M., van Eijk, K.R., Walters, R.K., Westlye, L.T., Whelan, C.D., Winkler, A.M., Zwiers, M.P., Alhusaini, S., Athanasiu, L., Ehrlich, S., Hakobjan, M.M., Hartberg, C.B., Haukvik, U.K., Heister, A.J., Hoehn, D., Kasperaviciute, D., Liewald, D.C., Lopez, L.M., Makkinje, R.R., Matarin, M., Naber, M.A., McKay, D.R., Needham, M., Nugent, A.C., Putz, B., Royle, N.A., Shen, L., Sprooten, E., Trabzuni, D., van der Marel, S.S., van Hulzen, K.J., Walton, E., Wolf, C., Almasy, L., Ames, D., Arepalli, S., Assareh, A.A., Bastin, M.E., Brodaty, H., Bulayeva, K.B., Carless, M.A., Cichon, S., Corvin, A., Curran, J.E., Czisch, M., de Zubicaray, G.I., Dillman, A., Duggirala, R., Dyer, T.D., Erk, S., Fedko, I.O., Ferrucci, L., Foroud, T.M., Fox, P.T., Fukunaga, M., Gibbs, J.R., Goring, H.H., Green, R.C., Guelfi, S., Hansell, N.K., Hartman, C.A., Hegenscheid, K., Heinz, A., Hernandez, D.G., Heslenfeld, D.J., Hoekstra, P.J., Holsboer, F., Homuth, G., Hottenga, J.J., Ikeda, M., Jack Jr., C.R., Jenkinson, M., Johnson, R., Kanai, R., Keil, M., Kent Jr., J.W., Kochunov, P., Kwok, J.B., Lawrie, S.M., Liu, X., Longo, D.L., McMahon, K.L., Meisenzahl, E., Melle, I., Mohnke, S., Montgomery, G.W., Mostert, J.C., Muhleisen, T.W., Nalls, M.A., Nichols, T.E., Nilsson, L.G., Nothen, M.M., Ohi, K., Olvera, R.L., Perez-Iglesias, R., Pike, G.B., Potkin, S.G., Reinvang, I., Reppermund, S., Rietschel, M., Romanczuk-Seiferth, N., Rosen, G.D., Rujescu, D., Schnell, K., Schofield, P.R., Smith, C., Steen, V.M., Sussmann, J.E., Thalamuthu, A., Toga, A.W., Traynor, B.J., Troncoso, J., Turner, J.A., Valdes Hernandez, M.C., van 't Ent, D., van der Brug, M., van der Wee, N.J., van Tol, M.J., Veltman, D.J., Wassink, T.H., Westman, E., Zielke, R.H., Zonderman, A.B., Ashbrook, D.G., Hager, R., Lu, L., McMahon, F.J., Morris, D.W., Williams, R.W., Brunner, H.G., Buckner, R.L., Buitelaar, J.K., Cahn, W., Calhoun, V.D., Cavalleri, G.L., Crespo-Facorro, B., Dale, A.M., Davies, G.E., Delanty, N., Depondt, C., Djurovic, S., Drevets, W.C., Espeseth, T., Gollub, R.L., Ho, B.C., Hoffmann, W., Hosten, N., Kahn, R.S., Le Hellard, S., Meyer-Lindenberg, A., Muller-Myhsok, B., Nauck, M., Nyberg, L., Pandolfo, M., Penninx, B.W., Roffman, J.L., Sisodiya, S.M., Smoller, J.W., van Bokhoven, H., van Haren, N.E., Volzke, H., Walter, H., Weiner, M.W., Wen, W. White, T., Agartz, I., Andreassen, O.A., Blangero, J., Boomsma, D.I., Brouwer, R.M., Cannon, D.M., Cookson, M.R., de Geus, E.J., Deary, I.J., Donohoe, G., Fernandez, G., Fisher, S.E., Francks, C., Glahn, D.C., Grabe, H.J., Gruber, O., Hardy, J., Hashimoto, R., Hulshoff Pol, H.E., Jonsson, E.G., Kloszewska, I., Lovestone, S., Mattay, V.S., Mecocci, P., McDonald, C., McIntosh, A.M., Ophoff, R.A., Paus, T., Pausova, Z., Ryten, M., Sachdev, P.S., Saykin, A.J., Simmons, A., Singleton, A., Soininen, H., Wardlaw, J.M. Weale, M.E., Weinberger, D.R., Adams, H.H., Launer, L.J., Seiler, S., Schmidt, R., Chauhan, G., Satizabal, C.L., Becker, J.T., Yanek, L., van der Lee, S.J., Ebling, M., Fischl, B., Longstreth Jr., W.T., Greve, D., Schmidt, H., Nyquist, P., Vinke, L.N., van Duijn, C.M., Xue, L., Mazoyer, B., Bis, J.C., Gudnason, V., Seshadri, S., Ikram, M.A., Alzheimer's Disease Neuroimaging Initiative, CHARGE Consortium, EPIGEN IMAGEN, SYS, Martin, N.G., Wright, M.J., Schumann, G., Franke, B., Thompson, P.M., Medland, S.E., 2015. Common genetic variants influence human subcortical brain structures. Nature 520 (7546), 224-229.

Hill, S.K., Reilly, J.L., Keefe, R.S., Gold, J.M., Bishop, J.R., Gershon, E.S., Tamminga, C.A., Pearlson, G.D., Keshavan, M.S., Sweeney, J.A., 2013. Neuropsychological impairments in schizophrenia and psychotic bipolar disorder: Findings from the Bipolar-Schizophrenia Network on Intermediate Phenotypes (B-SNIP) study. Am. J. Psychiatry 170 (11), 1275-1284.

Holmes, A.J., Hollinshead, M.O., O'Keefe, T.M., Petrov, V.I., Fariello, G.R., Wald, L.L., Fischl, B., Rosen, B.R., Mair, R.W., Roffman, J.L., Smoller, J.W., Buckner, R.L., 2015. Brain Genomics Superstruct Project initial data release with structural, functional, and behavioral measures. Sci. Data 2, 150031.

Hubbard, L., Tansey, K.E., Rai, D., Jones, P., Ripke, S., Chambert, K.D., Moran, J.L., McCarroll, S.A., Linden, D.E., Owen, M.J., O'Donovan, M.C., Walters, J.T., Zammit, S., 2016. Evidence of common genetic overlap between schizophrenia and cognition. Schizophr. Bull. 42 (3), 832-842.

Kay, S.R., Fiszbein, A., Opler, L.A., 1987. The Positive And Negative Syndrome Scale (PANSS) for schizophrenia. Schizophr. Bull. 13 (2), 261-276.

Keshavan, M.S., Kulkarni, S., Bhojraj, T., Francis, A., Diwadkar, V., Montrose, D.M., Seidman, L.J., Sweeney, J., 2010. Premorbid cognitive deficits in young relatives of schizophrenia patients. Front. Hum. Neurosci. 3, 62.

Khandaker, G.M., Barnett, J.H., White, I.R., Jones, P.B., 2011. A quantitative meta-analysis of population-based studies of premorbid intelligence and schizophrenia. Schizophr. Res. 132 (2-3), 220-227.

Lee, P.H., Baker, J.T., Holmes, A.J., Jahanshad, N., Ge, T., Jung, J.Y., Cruz, Y., Manoach, D.S. Hibar, D.P., Faskowitz, J., McMahon, K.L., de Zubicaray, G.I., Martin, N.H., Wright, M.J., Öngür, D., Buckner, R., Roffman, J., Thompson, P.M., Smoller, J.W., 2016. Partitioning heritability analysis reveals a shared genetic basis of brain anatomy and schizophrenia. Mol. Psychiatry 21 (12), 1680-1689.

Lencz, T., Szeszko, P.R., DeRosse, P., Burdick, K.E., Bromet, E.J., Bilder, R.M., Malhotra, A.K. 2010. A schizophrenia risk gene, ZNF804A, influences neuroanatomical and neurocognitive phenotypes. Neuropsychopharmacol. Off. Publ. Am. Coll. Neuropsychopharmacol. 35 (11), 2284-2291.

Lencz, T., Knowles, E., Davies, G., Guha, S., Liewald, D.C., Starr, J.M., Djurovic, S., Melle, I., Sundet, K., Christoforou, A., Reinvang, I., Mukherjee, S., DeRosse, P., Lundervold, A., Steen, V.M., John, M., Espeseth, T., Raikkonen, K., Widen, E., Palotie, A., Eriksson, J.G., Giegling, I., Konte, B., Ikeda, M., Roussos, P., Giakoumaki, S., Burdick, K.E., Payton, A. Ollier, W., Horan, M., Donohoe, G., Morris, D., Corvin, A., Gill, M., Pendleton, N., Iwata, N., Darvasi, A., Bitsios, P., Rujescu, D., Lahti, J., Hellard, S.L., Keller, M.C., Andreassen, O.A., Deary, I.J., Glahn, D.C., Malhotra, A.K., 2014. Molecular genetic evidence for overlap between general cognitive ability and risk for schizophrenia: A report from the Cognitive Genomics consorTium (COGENT). Mol. Psychiatry 19 (2), 168-174.

Lewandowski, K.E., Cohen, B.M., Öngür, D., 2011. Evolution of neuropsychological dysfunction during the course of schizophrenia and bipolar disorder. Psychol. Med. 41 (2), 225-241.

Lichtenstein, P., Yip, B.H., Bjork, C., Pawitan, Y., Cannon, T.D., Sullivan, P.F., Hultman, C.M. 2009. Common genetic determinants of schizophrenia and bipolar disorder in Swedish families: A population-based study. Lancet 373 (9659), 234-239.

Liebers, D.T., Pirooznia, M., Seiffudin, F., Musliner, K.L., Zandi, P.P., Goes, F.S., 2016. Polygenic risk of schizophrenia and cognition in a population-based survey of older adults. Schizophr. Bull. 42 (4), 984-991.

Malhotra, D., Sebat, J., 2012. CNVs: Harbingers of a rare variant revolution in psychiatric genetics. Cell 148 (6), 1223-1241.

Martin, A.K., Robinson, G., Reutens, D., Mowry, B., 2015. Common genetic risk variants are associated with positive symptoms and decision-making ability in patients with schizophrenia. Psychiatry Res. 229 (1-2), 606-608.

Nuechterlein, K.H., Green, M.F., Kern, R.S., Baade, L.E., Barch, D.M., Cohen, J.D., Essock, S. Fenton, W.S., Frese 3rd, F.J., Gold, J.M., Goldberg, T., Heaton, R.K., Keefe, R.S., Kraemer, H., Mesholam-Gately, R., Seidman, L.J., Stover, E., Weinberger, D.R., Young, A.S., Zalcman, S., Marder, S.R., 2008. The MATRICS Consensus Cognitive Battery, part 1: Test selection, reliability, and validity. Am. J. Psychiatry 165 (2), 203-213.

Peralta, V., Cuesta, M.J., 1994. Psychometric properties of the positive and negative syndrome scale (PANSS) in schizophrenia. Psychiatry Res. 53 (1), 31-40.

PGC Schizophrenia Working Group, 2014. Biological insights from 108 schizophrenia-associated genetic loci. Nature 511 (7510), 421-427.

Psychosis Endophenotypes International Consortium, Wellcome Trust Case-Control Consortium, Bramon, E., Pirinen, M., Strange, A., Lin, K., Freeman, C., Bellenguez, C., Su, Z., Band, G., Pearson, R., Vukcevic, D., Langford, C., Deloukas, P., Hunt, S., Gray, E., Dronov, S., Potter, S.C., Tashakkori-Ghanbaria, A., Edkins, S., Bumpstead, S.J., Arranz, M.J., Bakker, S., Bender, S., Bruggeman, R., Cahn, W., Chandler, D., Collier, D.A., Crespo-Facorro, B., Dazzan, P., de Haan, L., Di Forti, M., Dragovic, M., Giegling, I. Hall, J., lyegbe, C., Jablensky, A., Kahn, R.S., Kalaydjieva, L., Kravariti, E., Lawrie, S., Linszen, D.H., Mata, I., McDonald, C., McIntosh, A., Myin-Germeys, I., Ophoff, R.A Pariante, C.M., Paunio, T., Picchioni, M., Psychiatric Genomics Consortium, Ripke, S. Rujescu, D., Sauer, H., Shaikh, M., Sussmann, J., Suvisaari, J., Tosato, S., Toulopoulou, T., Van Os, J., Walshe, M., Weisbrod, M., Whalley, H., Wiersma, D., Blackwell, J.M., Brown, M.A., Casas, J.P., Corvin, A., Duncanson, A., Jankowski, J.A., Markus, H.S., Mathew, C.G., Palmer, C.N., Plomin, R., Rautanen, A., Sawcer, S.J., Trembath, R.C., Wood, N.W., Barroso, I., Peltonen, L., Lewis, C.M., Murray, R.M., Donnelly, P., Powell, J., Spencer, C.C., 2014. A genome-wide association analysis of a broad psychosis phenotype identifies three loci for further investigation. Biol. Psychiatry 75 (5), 386-397.

Purcell, S., Neale, B., Todd-Brown, K., Thomas, L., Ferreira, M.A., Bender, D., Maller, J., Sklar, P., de Bakker, P.I., Daly, M.J., Sham, P.C., 2007. PLINK: A tool set for whole-genome association and population-based linkage analyses. Am. J. Hum. Genet. 81 (3), 559-575.

Purcell, S.M., Wray, N.R., Stone, J.L., Visscher, P.M., O'Donovan, M.C., Sullivan, P.F., Sklar, P. 2009. Common polygenic variation contributes to risk of schizophrenia and bipolar disorder. Nature 460 (7256), 748-752.

Ripke, S., O'Dushlaine, C., Chambert, K., Moran, J.L., Kahler, A.K., Akterin, S., Bergen, S.E. Collins, A.L., Crowley, J.J., Fromer, M., Kim, Y., Lee, S.H., Magnusson, P.K., Sanchez, N., Stahl, E.A., Williams, S., Wray, N.R., Xia, K., Bettella, F., Borglum, A.D., Bulik-Sullivan, B.K., Cormican, P., Craddock, N., de Leeuw, C., Durmishi, N., Gill, M., Golimbet, V. Hamshere, M.L., Holmans, P., Hougaard, D.M., Kendler, K.S., Lin, K., Morris, D.W. Mors, O., Mortensen, P.B., Neale, B.M., O'Neill, F.A., Owen, M.J., Milovancevic, M.P., Posthuma, D., Powell, J., Richards, A.L., Riley, B.P., Ruderfer, D., Rujescu, D. Sigurdsson, E., Silagadze, T., Smit, A.B., Stefansson, H., Steinberg, S., Suvisaari, J., Tosato, S., Verhage, M., Walters, J.T., Multicenter Genetic Studies of Schizophrenia Consortium, Levinson, D.F., Gejman, P.V., Kendler, K.S., Laurent, C., Mowry, B.J., O'Donovan, M.C., Owen, M.J., Pulver, A.E., Riley, B.P., Schwab, S.G., Wildenauer, D.B. Dudbridge, F., Holmans, P., Shi, J., Albus, M., Alexander, M., Campion, D., Cohen, D. Dikeos, D., Duan, J., Eichhammer, P., Godard, S., Hansen, M., Lerer, F.B., Liang, K.Y. Maier, W., Mallet, J., Nertney, D.A., Nestadt, G., Norton, N., O'Neill, F.A. Papadimitriou, G.N., Ribble, R., Sanders, A.R., Silverman, J.M., Walsh, D., Williams, N.M., Wormley, B., Psychosis Endophenotypes International Consortium, Arranz, M.J., Bakker, S., Bender, S., Bramon, E., Collier, D., Crespo-Facorro, B., Hall, J., Iyegbe, C., Jablensky, A., Kahn, R.S., Kalaydjieva, L., Lawrie, S., Lewis, C.M., Lin, K., Linszen, D.H., Mata, I., McIntosh, A., Murray, R.M., Ophoff, R.A., Powell, J., Rujescu, D., Van Os, J., Walshe, M., Weisbrod, M., Wiersma, D., Wellcome Trust Case Control Consortium, Donnelly, P., Barroso, I., Blackwell, J.M., Bramon, E., Brown, M.A., Casas, J.P., Corvin, A.P., Deloukas, P., Duncanson, A., Jankowski, J., Markus, H.S., Mathew C.G., Palmer, C.N., Plomin, R., Rautanen, A., Sawcer, S.J., Trembath, R.C. Viswanathan, A.C., Wood, N.W., Spencer, C.C., Band, G., Bellenguez, C., Freeman, C., Hellenthal, G., Giannoulatou, E., Pirinen, M., Pearson, R.D., Strange, A., Su, Z., Vukcevic, D., Donnelly, P., Langford, C., Hunt, S.E., Edkins, S., Gwilliam, R., Blackburn, H., Bumpstead, S.J., Dronov, S., Gillman, M., Gray, E., Hammond, N., Jayakumar, A. McCann, O.T., Liddle, J., Potter, S.C., Ravindrarajah, R., Ricketts, M., TashakkoriGhanbaria, A., Waller, M.J., Weston, P., Widaa, S., Whittaker, P., Barroso, I., Deloukas, 
P., Mathew, C.G., Blackwell, J.M., Brown, M.A., Corvin, A.P., McCarthy, M.I., Spencer, C.C., Bramon, E., Corvin, A.P., O'Donovan, M.C., Stefansson, K., Scolnick, E., Purcell, S., McCarroll, S.A., Sklar, P., Hultman, C.M., Sullivan, P.F., 2013. Genome-wide association analysis identifies 13 new risk loci for schizophrenia. Nat. Genet. 45 (10), 1150-1159.

Robinson, E.B., Kirby, A., Ruparel, K., Yang, J., McGrath, L., Anttila, V., Neale, B.M., Merikangas, K., Lehner, T., Sleiman, P.M., Daly, M.J., Gur, R., Gur, R., Hakonarson, H., 2015. The genetic architecture of pediatric cognitive abilities in the Philadelphia Neurodevelopmental Cohort. Mol. Psychiatry 20 (4), 454-458.

Segall, J.M., Turner, J.A., van Erp, T.G., White, T., Bockholt, H.J., Gollub, R.L., Ho, B.C. Magnotta, V., Jung, R.E., McCarley, R.W., Schulz, S.C., Lauriello, J., Clark, V.P., Voyvodic, J.T., Diaz, M.T., Calhoun, V.D., 2009. Voxel-based morphometric multisite collaborative study on schizophrenia. Schizophr. Bull. 35 (1), 82-95.

Seidman, L.J., Hellemann, G., Nuechterlein, K.H., Greenwood, T.A., Braff, D.L., Cadenhead, K.S., Calkins, M.E., Freedman, R., Gur, R.E., Gur, R.C., Lazzeroni, L.C., Light, G.A. Olincy, A., Radant, A.D., Siever, L.J., Silverman, J.M., Sprock, J., Stone, W.S., Sugar, C., Swerdlow, N.R., Tsuang, D.W., Tsuang, M.T., Turetsky, B.I., Green, M.F., 2015. Facto structure and heritability of endophenotypes in schizophrenia: Findings from the Consortium on the Genetics of Schizophrenia (COGS-1). Schizophr. Res. 163 (1-3), 73-79.

Shenton, M.E., Dickey, C.C., Frumin, M., McCarley, R.W., 2001. A review of MRI findings in schizophrenia. Schizophr. Res. 49 (1-2), 1-52.

Spearman, C., 1904. General intelligence objectively determined and measured. Am. J. Psychol. 15, 201-293.

Stone, W.S., Seidman, L.J., 2016. Neuropsychological and structural neuroimaging endophenotypes in schizophrenia. In: Cicchetti, D. (Ed.), Developmental Psychopathology, 3 ed. John Wiley \& Sons, Inc., Hoboken, New Jersey, pp. 931-965.

Sudmant, P.H., Rausch, T., Gardner, E.J., Handsaker, R.E., Abyzov, A., Huddleston, J., Zhang Y., Ye, K., Jun, G., Hsi-Yang Fritz, M., Konkel, M.K., Malhotra, A., Stutz, A.M., Shi, X., Paolo Casale, F., Chen, J., Hormozdiari, F., Dayama, G., Chen, K., Malig, M., Chaisson, M.J., Walter, K., Meiers, S., Kashin, S., Garrison, E., Auton, A., Lam, H.Y., Jasmine Mu, X., Alkan, C., Antaki, D., Bae, T., Cerveira, E., Chines, P., Chong, Z., Clarke, L., Dal, E. Ding, L., Emery, S., Fan, X., Gujral, M., Kahveci, F., Kidd, J.M., Kong, Y., Lameijer, E.W. McCarthy, S., Flicek, P., Gibbs, R.A., Marth, G., Mason, C.E., Menelaou, A., Muzny, D.M., Nelson, B.J., Noor, A., Parrish, N.F., Pendleton, M., Quitadamo, A., Raeder, B. Schadt, E.E., Romanovitch, M., Schlattl, A., Sebra, R., Shabalin, A.A., Untergasser, A Walker, J.A., Wang, M., Yu, F., Zhang, C., Zhang, J., Zheng-Bradley, X., Zhou, W., Zichner, T., Sebat, J., Batzer, M.A., McCarroll, S.A., 1000 Genomes Project Consortium, Mills, R.E., Gerstein, M.B., Bashir, A., Stegle, O., Devine, S.E., Lee, C. Eichler, E.E., Korbel, J.O., 2015. An integrated map of structural variation in 2,504 human genomes. Nature 526 (7571), 75-81.

Sullivan, P.F., Kendler, K.S., Neale, M.C., 2003. Schizophrenia as a complex trait: Evidence from a meta-analysis of twin studies. Arch. Gen. Psychiatry 60 (12), 1187-1192.

Tamminga, C.A., Ivleva, E.I., Keshavan, M.S., Pearlson, G.D., Clementz, B.A., Witte, B. Morris, D.W., Bishop, J., Thaker, G.K., Sweeney, J.A., 2013. Clinical phenotypes of psychosis in the Bipolar-Schizophrenia Network on Intermediate Phenotypes (B-SNIP). Am. J. Psychiatry 170 (11), 1263-1274.

The 1000 Genomes Project Consortium, Auton, A., Brooks, L.D., Durbin, R.M., Garrison, E.P., Kang, H.M., Korbel, J.O., Marchini, J.L., McCarthy, S., McVean, G.A., Abecasis, G.R., 2015. A global reference for human genetic variation. Nature 526 (7571), 68-74.

Thermenos, H.W., Keshavan, M.S., Juelich, R.J., Molokotos, E., Whitfield-Gabrieli, S., Brent, B.K., Makris, N., Seidman, L.J., 2013. A review of neuroimaging studies of young relatives of individuals with schizophrenia: A developmental perspective from schizotaxia to schizophrenia. Am. J. Med. Genet. B Neuropsychiatr. Genet. 162B (7), 604-635.

Toulopoulou, T., Goldberg, T.E., Mesa, I.R., Picchioni, M., Rijsdijk, F., Stahl, D., Cherny, S.S., Sham, P., Faraone, S.V., Tsuang, M., Weinberger, D.R., Seidman, L.J., Murray, R.M. 2010. Impaired intellect and memory: A missing link between genetic risk and schizophrenia? Arch. Gen. Psychiatry 67 (9), 905-913.

Trampush, J.W., Yang, M.L., Yu, J., Knowles, E., Davies, G., Liewald, D.C., Starr, J.M., Djurovic S., Melle, I., Sundet, K., Christoforou, A., Reinvang I., DeRosse, P., Lundervold, A. . Steen, V.M., Espeseth, T., Raikkonen, K., Widen, E., Palotie, A., Eriksson, J.G., Giegling,
I., Konte, B., Roussos, P., Giakoumaki, S., Burdick, K.E., Payton, A., Ollier, W., Horan, M., Chiba-Falek, O., Attix, D.K., Need, A.C., Cirulli, E.T., Voineskos, A.N., Stefanis, N.C., Avramopoulos, D., Hatzimanolis, A., Arking, D.E., Smyrnis, N., Bilder, R.M., Freimer, N.A., Cannon, T.D., London, E., Poldrack, R.A., Sabb, F.W., Congdon, E., Conley, E.D., Scult, M.A., Dickinson, D., Straub, R.E., Donohoe, G., Morris, D., Corvin, A., Gill, M., Hariri, A.R., Weinberger, D.R., Pendleton, N., Bitsios, P., Rujescu, D., Lahti, J., Le Hellard, S., Keller, M.C., Andreassen, O.A., Deary, I.J., Glahn, D.C., Malhotra, A.K., Lencz, T., 2017. GWAS meta-analysis reveals novel loci and genetic correlates for general cognitive function: A report from the COGENT consortium. Mol. Psychiatry 22 (3), 336-345

van Erp, T.G., Preda, A., Nguyen, D., Faziola, L., Turner, J., Bustillo, J., Belger, A., Lim, K.O., McEwen, S., Voyvodic, J., Mathalon, D.H., Ford, J., Potkin, S.G., FBIRN, 2014. Converting positive and negative symptom scores between PANSS and SAPS/SANS. Schizophr. Res. 152 (1), 289-294.

van Erp, T.G., Hibar, D.P., Rasmussen, J.M., Glahn, D.C., Pearlson, G.D., Andreassen, O.A Agartz, I., Westlye, L.T., Haukvik, U.K., Dale, A.M., Melle, I., Hartberg, C.B., Gruber, O., Kraemer, B., Zilles, D., Donohoe, G., Kelly, S., McDonald, C., Morris, D.W., Cannon, D.M., Corvin, A., Machielsen, M.W., Koenders, L., de Haan, L., Veltman, D.J., Satterthwaite, T.D., Wolf, D.H., Gur, R.C., Gur, R.E., Potkin, S.G., Mathalon, D.H., Mueller, B.A., Preda, A., Macciardi, F., Ehrlich, S., Walton, E., Hass, J., Calhoun, V.D., Bockholt, H.J., Sponheim, S.R., Shoemaker, J.M., van Haren, N.E., Pol, H.E., Ophoff, R.A., Kahn, R.S., Roiz-Santianez, R., Crespo-Facorro, B., Wang, L., Alpert, K.I., Jonsson, E.G., Dimitrova, R., Bois, C., Whalley, H.C., McIntosh, A.M., Lawrie, S.M., Hashimoto, R., Thompson, P.M., Turner, J.A., 2016. Subcortical brain volume abnormalities in 2028 individuals with schizophrenia and 2540 healthy controls via the ENIGMA consortium. Mol. Psychiatry 21 (4), 547-553.

van Scheltinga, A.F., Bakker, S.C., van Haren, N.E., Derks, E.M., Buizer-Voskamp, J.E., Cahn, W., Ripke, S., Psychiatric Genome-Wide Association Study Consortium, Ophoff, R.A., Kahn, R.S., 2013. Schizophrenia genetic variants are not associated with intelligence. Psychol. Med. 43 (12), 2563-2570.

Visscher, P.M., Hemani, G., Vinkhuyzen, A.A., Chen, G.B., Lee, S.H., Wray, N.R., Goddard, M.E., Yang, J., 2014. Statistical power to detect genetic (co)variance of complex traits using SNP data in unrelated samples. PLoS Genet. 10 (4), e1004269.

Walton, E., Geisler, D., Lee, P.H., Hass, J., Turner, J.A., Liu, J., Sponheim, S.R., White, T., Wassink, T.H., Roessner, V., Gollub, R.L., Calhoun, V.D., Ehrlich, S., 2013a. Prefrontal inefficiency is associated with polygenic risk for schizophrenia. Schizophr. Bull. 40 (6), 1263-1271.

Walton, E., Turner, J., Gollub, R.L., Manoach, D.S., Yendiki, A., Ho, B.C., Sponheim, S.R., Calhoun, V.D., Ehrlich, S., 2013b. Cumulative genetic risk and prefrontal activity in patients with schizophrenia. Schizophr. Bull. 39 (3), 703-711.

Wassink, T.H., Epping, E.A., Rudd, D., Axelsen, M., Ziebell, S., Fleming, F.W., Monson, E., Ho, B.C., Andreasen, N.C., 2012. Influence of ZNF804a on brain structure volumes and symptom severity in individuals with schizophrenia. Arch. Gen. Psychiatry 69 (9), 885-892.

Wilke, M., Rose, D.F., Holland, S.K., Leach, J.L., 2014. Multidimensional morphometric 3D MRI analyses for detecting brain abnormalities in children: Impact of control population. Hum. Brain Mapp. 35 (7), 3199-3215.

Woodberry, K.A., Giuliano, A.J., Seidman, L.J., 2008. Premorbid IQ in schizophrenia: A meta-analytic review. Am. J. Psychiatry 165 (5), 579-587.

Woods, S.W., 2003. Chlorpromazine equivalent doses for the newer atypical antipsychotics. J. Clin. Psychiatry 64 (6), 663-667.

Yeo, R.A., Gangestad, S.W., Walton, E., Ehrlich, S., Pommy, J., Turner, J.A., Liu, J., Mayer, A.R., Schulz, S.C., Ho, B.C., Bustillo, J.R., Wassink, T.H., Sponheim, S.R., Morrow, E.M. Calhoun, V.D., 2014. Genetic influences on cognitive endophenotypes in schizophrenia. Schizophr. Res. 156 (1), 71-75.

Zuk, O., Schaffner, S.F., Samocha, K., Do, R., Hechter, E., Kathiresan, S., Daly, M.J., Neale, B.M., Sunyaev, S.R., Lander, E.S., 2014. Searching for missing heritability: Designing rare variant association studies. Proc. Natl. Acad. Sci. U. S. A. 111 (4), E455-464. 